\title{
An HDLC Protocol Specification and Its Verification Using Image Protocols
}

\author{
A. UDAYA SHANKAR and SIMON S. LAM \\ University of Texas at Austin
}

We use an event-driven process model to specify a version of the High-Level Data Link Control (HDLC) protocol between two communicating protocol entities. The protocol is verified using the method of projections. The verification serves as a rigorous exercise to demonstrate the applicability of this method to the analysis of real-life communication protocols.

The HDLC protocol has two characteristics found in most real-life communication protocols. First, the HDLC protocol operates under real-time constraints that are important not only for its performance but also for its correct logical behavior. We specify this real-time behavior using time variables and time events. Second, the HDLC protocol has three distinguishable functions: connection management, and one-way data transfers between the protocol entities. For each of these functions, we construct an image protocol using the method of projections. With each image protocol we obtain inductively complete invariant assertions that state various desirable logical safety properties. From the properties of image protocols it follows that these safety properties as proved for the image protocols are also satisfied by the HDLC protocol presented herein. We also suggest a minor modification to HDLC that will make it well-structured.

Categories and Subject Descriptors: C.2.2 [Computer-Communication Networks]: Network Protocols-protocol architecture, HDLC, protocol verification; C.3 [Computer Systems Organization]: Special-Purpose and Application-Based Systems-real-time systems; D.2.4 [Software Engineering]: Program Verification-correctness proofs; F.3.1 [Logics and Meanings of Programs]: Specifying and Verifying and Reasoning About Programs-assertions, invariants, pre-and post-conditions, specification techniques

General Terms: Algorithms, Languages, Verification

Additional Key Words and Phrases: Communication protocols, data link control, HDLC protocol, method of projections, image protocols, message-passing networks, communicating processes

\section{INTRODUCTION}

The High-Level Data Link Control (HDLC) protocol corresponds to a layer 2 protocol within the OSI reference model $[7,8,9,23]$. It is intended to provide reliable full-duplex data transfer between layer 3 protocol entities, using errorprone physical communication channels of layer 1 . The specification of HDLC in the ISO documents precisely defines low-level protocol functions, such as error

This work was supported by NSF Grants ECS78-01803 and ECS83-04734.

This paper was submitted for publication in Transactions on Computer Systems by the authors and not by the SIGCOMM Symposium Program Committee. Author's addresses: A. U. Shankar, Department of Computer Science, University of Maryland, College Park, MD 20742; S. S. Lam, Department of Computer Sciences, University of Texas at Austin, Austin, TX 78712.

Permission to copy without fee all or part of this material is granted provided that the copies are not made or distributed for direct commercial advantage, the ACM copyright notice and the title of the publication and its date appear, and notice is given that copying is by permission of the Association for Computing Machinery. To copy otherwise, or to republish, requires a fee and/or specific permission.

(C) 1983 ACM 0734-2071/83/1100-0331 $\$ 00.75$

ACM Transactions on Computer Systems, Vol. 1, No. 4, November 1983, Pages 331-368. 


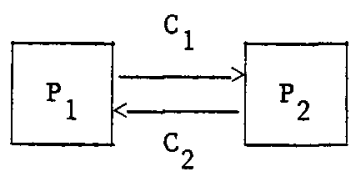

Fig. 1. The protocol system model.

detection and frame synchronization. Formats of three types of frames specifying the encoding of control and data messages are also clearly defined. Aside from these basic definitions, however, the HDLC documents leave many options to be decided by the protocol implementor. In particular, one can choose from a variety of data link configurations and three operational modes that specify balanced or unequal relationships among the communicating entities. Also, various subsets of the messages can be used, instead of the entire set defined. Further, some aspects of HDLC are described informally in English and are not rigorously specified.

In this paper, we use an event-driven process model to specify a version of the HDLC protocol, and then apply the method of projections to verify it $[13,16$, $20]$. This verification serves as an exercise for demonstrating the applicability of this method (see Figure 1). $P_{1}$ is a primary HDLC entity and $P_{2}$ a secondary HDLC entity operating in the Asynchronous Response Mode (ARM). $C_{1}$ and $C_{2}$ are unreliable communication channels. Our protocol uses the basic repertoire of HDLC commands and responses (with the exception of the CMDR response). It includes the use of poll/final cycles for checkpointing and connection management, timers for timeouts, cyclic sequence numbers and sliding windows of size $N$ for data transfers, and ready/not ready messages for flow control [8]. Our protocol incorporates all of the principal HDLC functions.

\subsection{Analysis of Multifunction Protocols}

The HDLC protocol has at least three distinguishable functions: connection management, and one-way data transfers in two directions. A multifunction protocol such as HDLC is very complex and cannot be easily analyzed. To reduce the complexity of analysis, an approach that appears attractive is to decompose each protocol entity into modules for handling the different functions of the protocol. For example, each protocol entity in HDLC may be decomposed into three functional modules as shown in Figure 2. Each module communicates with a corresponding module in the other protocol entity to accomplish one of the three functions. Bochmann and Chung [2] used a decomposition approach to specify a version of the HDLC protocol. However, the decomposition approach does not seem to facilitate analysis of the protocol. The main difficulty is that significant interaction exists among the modules. We identify two types of dependencies. First, modules interact through shared variables within an entity. Second, they also interact because data and control messages sent by different modules in one entity to their respective modules in the other entity are typically encoded in the same protocol message (shared protocol messages).

Most communication protocols that have been rigorously analyzed and presented in the literature are concerned with a single function: either a connection management function $[1,10,14]$ or a one-way data transfer function $[22,6,4]$. For example, both safety and liveness properties have been formulated and proved for Stenning's protocol [22,6]. Stenning's protocol is a one-way data transfer protocol. It corresponds to the interaction of a data send module and a 


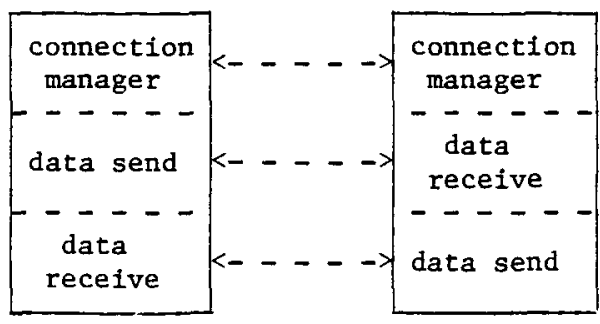

Fig. 2. Functional decomposition of HDLC.

data receive module in isolation (see Figure 2). Any interaction between these modules and other modules is not accounted for. As such, this protocol constitutes just one function of a real-life protocol such as HDLC. The following question arises: Are the safety and liveness properties that are proved for the one-way data transfer protocol still valid when it is implemented as part of a multifunction protocol with the two types of dependencies mentioned above?

We use the method of projections $[11,12,13,16,20]$ to break up our HDLC protocol analysis problem into smaller problems. The theory of projections is described in [13] and [16]; we will not go into its details here. The projection method is different from the straightforward approach of decomposing protocol entities into functional modules. The objective is to construct from the given HDLC protocol an image protocol for each of the three functions that are of interest to us (referred to as the projected functions).

An image protocol is specified just like any real protocol. The states, messages and events of entities in an image protocol are obtained by treating groups of states, messages and events in the original protocol as equivalent and aggregating them. As a result, an image protocol is smaller than the original protocol. Any safety property that holds for the image protocol also holds for the original protocol. Additionally, if an image protocol satisfies a well-formed property then it is faithful: Any logical property, safety or liveness, that can be stated for the image protocol holds in the image protocol if and only if it also holds in the original protocol. (Fairness in the scheduling of enabled events in the original protocol system is assumed; that is, no enabled event will be indefinitely delayed [15].)

The objective of our construction procedure is to generate the smallest image protocol that is of sufficient resolution to verify a desired logical property $A_{0}$ of the projected function. For example, the image protocol that is constructed for HDLC connection management is similar to a handshake protocol [1]. The image protocol for HDLC one-way data transfer is similar to other one-way data transfer protocols based on a sliding window mechanism, but is augmented with initialization and checkpointing features. There are two methods that we use to determine the desired resolution. The first method is applicable when $A_{0}$ is a safety (including real-time) property. The second method constructs a wellformed image protocol, and is applicable whether $A_{0}$ is a safety or liveness property. In each method, an initial resolution derived from $A_{0}$ is successively refined.

The construction of well-formed image protocols involves an examination of protocol entities individually. There is no need to examine the global reachability 
space of the image protocol interaction or of the original protocol interaction. Given a multifunction protocol (such as HDLC), a well-formed image protocol can always be obtained for each function by increasing its resolution. However, the successful construction of well-formed image protocols that are much smaller than the original multifunction protocol depends upon whether the multifunction protocol has a good structure. Thus, one can think of a multifunction protocol as being well-structured if it possesses small well-formed image protocols for its functions.

\subsection{Real-Time Behavior}

Another important characteristic of real-life communication protocols is that they are time-dependent systems. Real-time constraints (such as bounded response times, packet lifetimes, etc.) exist within individual entities and channels [21]. These local time constraints give rise to global precedence relations between remote events. Such global relations are essential to the correct functioning of the protocol system. The modeling of real-time behavior has usually been neglected in previous protocol analyses.

To verify a time-dependent system, it is necessary to include measures of real time in the modeling of the protocol system. If time is not modeled explicitly, then one is forced to resort to informal arguments about global timing relations in the system. Such informal arguments are inadequate and are the source of many protocol system design errors [3].

The real-time behavior of communication protocols has implications regarding the formulation of liveness assertions. Typically, if a protocol does not achieve progress (transfer of data, establishment of a connection, etc.) within a bounded time duration $T$, then the protocol resorts to some alternative action (abort, reset, retransmission, etc.). The protocol will not wait for a finite but unbounded amount of time. Hence, a temporal logic liveness assertion [6] such as "eventually, a data block will be transferred" is not realistic. More appropriate is a real-time specification such as "if within a time duration $T$ the data block is not transferred then at least $n$ retransmissions of the data block have occurred, all of which failed, and the protocol has reset."

We model measures of time by incorporating time variables and time events into our protocol system model [17]. With time variables and time events, the real-time behavior of communication protocols can be stated by safety assertions; temporal logic liveness assertions are not needed.

\subsection{Summary of This Paper}

In Section 2, we first describe an event-driven process model of a protocol system. Each component (entity or channel) of the protocol system is modeled as an event-driven process that manipulates a set of variables local to itself and interacts with adjacent components by message passing. The model includes several realistic protocol features such as multifield messages and the use of timers. This model is then used to specify the HDLC protocol.

In Section 3, we apply the method of projections to verify the HDLC protocol. In Section 3.1, we outline the definition of image protocols and two methods for obtaining image protocols. In Sections 3.2, 3.3 and 3.4, we construct from the given HDLC protocol an image protocol for each of the three functions that are 
of interest to us. For each image protocol, we obtain invariant safety assertions concerning some desired logical behavior of the projected function. From the properties of image protocols these assertions also hold for the entire HDLC protocol.

Of the three image protocols obtained, the image protocol for connection management is well-formed. However, the image protocols for the one-way data transfers are not well-formed. In order for these data transfer image protocols to be well-formed, they have to be made substantially larger to account for dependencies in the HDLC protocol between the two one-way data transfer functions.

In Section 3.5, we describe a minor modification to the HDLC protocol that allows small well-formed image protocols to be constructed for each of the oneway data transfer functions, as well as for the connection management function. The invariant safety assertions obtained in Sections 3.2, 3.3 and 3.4 continue to hold for the connection management and data transfer image protocols of the modified HDLC protocol. The HDLC protocol with this modification can be regarded as a well-structured protocol.

\section{AN HDLC/ARM PROTOCOL}

In this section, we describe the HDLC/ARM protocol for two protocol entities. ARM denotes the Asynchronous Response Mode of operation. Let $P_{1}$ be the primary HDLC entity, and let $P_{2}$ be the secondary HDLC entity. $P_{1}$ sends messages to $P_{2}$ using channel $C_{1}$, and $P_{2}$ sends messages to $P_{1}$ using channel $C_{2}$ (see Figure 1). There is a user at entity $P_{1}$ and a user at entity $P_{2}$. The HDLC protocol system offers the users a reliable connection that (a) can be opened/ closed by the user at $\mathrm{P}_{1}$, and (b) when open, allows each user to send data blocks to the other user in sequence (without loss, duplication or reordering).

\subsection{Assumptions about the Environment}

To obtain assertions about the logical behavior of the protocol system, a few assumptions are needed about the environment in which HDLC operates. At any time, channel $\mathrm{C}_{i}$ contains a (possibly empty) sequence of messages sent by $\mathrm{P}_{i}$, for $i=1$ and $i=2$. Messages in the channels may be corrupted by noise, but not reordered or duplicated. When $\mathrm{P}_{i}$ sends a message, that message is appended to the tail of the message sequence in $\mathrm{C}_{i}$. When channel $\mathrm{C}_{i}$ is not empty, the first message (at the head of the message sequence) can be removed and passed on to $\mathrm{P}_{j}(j \neq i)$, provided that the message is not corrupted. If the message is corrupted, it is deleted and not passed on to $\mathrm{P}_{j}$ (we assume a perfect error-detection mechanism). The frame-level functions of HDLC [7], such as the frame formatting of HDLC messages, bit insertion/deletion to make flags unique, error detecting, etc., are not considered as part of the entities $P_{1}$ and $P_{2}$, but have been included in the channel model. Finally, messages in the channels have a bounded lifetime. The first message in channel $\mathrm{C}_{i}$ is deleted if it has been in the channel for a specified time, denoted by MaxDelay . $_{\text {. }}$

\subsection{Event-Driven Process Model}

Each component of the protocol system (i.e., protocol entity or channel) is modeled as an event-driven process that manipulates a set of variables local to 
itself and interacts with adjacent components by message passing. The events of an entity consist of message sends, message receptions and changes internal to the entity. The events of a channel correspond to transformations on the channel message sequence. An event can occur only if variables of the protocol system satisfy certain conditions, referred to as the enabling condition of the event. When an enabled event occurs, variables of the protocol system are affected. Whenever an event-driven process has enabled events, any one of them can occur.

2.2.1. Time Variables and Time Events. In HDLC, each protocol entity guarantees certain constraints on the time intervals between occurrences of events involving that entity. Also, recall that messages in channels have bounded lifetimes. Because (physical) time elapses at the same rate everywhere, these time constraints give rise to precedence relations between remote events in different components. Furthermore, these precedence relations are vital to the proper functioning of the HDLC protocol. We cannot adequately model such a time-dependent system by using only entity and channel events. It is necessary to relate the elapsed times measured at different components. We do this by introducing time variables in the components to measure elapsed time in integer ticks, and time events to age the time variables $[16,17]$.

Each time variable takes its values from $N_{t}=\{$ Off, $0,1,2, \ldots\}$. A time variable is termed inactive if its value is Off; otherwise it is termed active. The value of a time variable can be changed in only two ways. First, it can be aged by a time event. When an active time variable is aged its value is incremented by 1 ; when an inactive time variable is aged its value is not affected. Second, a time variable in a component can be reset to any value in $N_{t}$ by a system event involving that component. Thus, for an active time variable, the difference between its current value and the value it was last reset to indicates the time elapsed since the last reset.

We will use two types of time variables in our model: global time variables and local time variables. All global time variables in a system model are aged by the same time event, referred to as the global time event. Thus, all active global time variables are coupled. The global time event models the elapse of physical time in the protocol system model. Global time variables are typically used to model time constraints that are satisfied by components without the use of timers.

Local time variables are used to model the timers that are implemented in system components. To each local time variable $t$ there is a unique local time event that ages $t$ (and $t$ alone). Thus, $t$ is not directly coupled to any other time variable. To specify its accuracy, we associate with $t$ a global time variable $t^{*}$ and a reset value $t_{0}$. Whenever $t$ is reset, both $t^{*}$ and $t_{0}$ are reset to the same value. $t^{*}$ is affected by the global time event like any other global time variable. The accuracy of local time variable $t$ is specified by its accuracy axiom which bounds $t-t^{*}$ at any time. For example, the accuracy axiom $\left|t-t^{*}\right| \leq 1+a\left(t^{*}\right.$ $\left.-t_{0}\right)$ can specify a timer with maximum relative error $a$ in its clock frequency (Off - Off is treated as 0 ).

In this model, neither the local time event of $t$ nor the global time event can occur if such an occurrence would violate the accuracy axiom. By placing additional constraints on the set of allowed values for time variables, other types of time constraints satisfied by a component can be modeled. For example, let $t$ 
be a time variable that is reset to 0 by event $e_{1}$ and reset to Off by event $e_{2}$. Let $D$ be a specified delay. Then, to model the time constraint that $e_{2}$ occurs no later than $D$ time units since the occurrence of $e_{1}$, we include $(t<D)$ in the enabling condition of the time event of $t$. Such constraints on time events are known as time axioms. (For a more detailed presentation, the reader is referred to [16] and [17].)

2.2.2. Messages of the Protocol Model. The messages of the protocol system have multiple fields, and are specified in terms of message types. A message type is specified by a tuple of the form $\left(M, F_{1}, F_{2}, \ldots, F_{n}\right)$, where $n \geq 0$. The first component contains the name of the message type and is a constant. The other components (if any) are the fields of the message type. Each field is a parameter that can take values from a specified set. The messages sent by each entity are specified by a list of such message types. For simplicity, we often use $\mathbf{M}$ to refer to $\left(M, F_{1}, F_{2}, \ldots, F_{n}\right)$.

2.2.3. Variables of the Entities and Channels. Each protocol entity has a set of variables, each with a specified domain of values. Some of these variables can be auxillary variables used only in specification/verification of the protocol system. Also, some of these variables can be time variables used in modeling time constraints satisfied by the entity.

In channel $\mathrm{C}_{i}$, we associate with every message in transit a global time value that indicates the time spent by that message in the channel. This time value is referred to as the age of the message. For channel $\mathrm{C}_{i}$, we define Channel Cas $_{i}$ the variable that represents, at any time, the sequence of (message, age) pairs in $C_{i}$.

2.2.4. Events of the Protocol Model. The events of the protocol system model can be categorized into entity events, channel events, and time events. We will describe them in that order. There are three types of entity events. We describe these events for entity $\mathrm{P}_{i}$.

1. For each message type $\left(M, F_{1}, \ldots, F_{n}\right)$ sent by $\mathrm{P}_{i}$, there is a Send_ $M$ event. This event is enabled if the values of the variables of $P_{i}$ satisfy a specified enabling condition predicate. Its occurrence appends an $M$-type message $\left(M, f_{1}, \ldots, f_{n}\right)$ to the tail of Channel $l_{i}$, and updates the values of the variables of $\mathrm{P}_{i}$ (where $f_{k}$ is an allowed value of $F_{k}$ ).

2. For each message type $\left(M, F_{1}, \ldots, F_{n}\right)$ sent by $\mathrm{P}_{j}(j \neq i)$, there is a $\operatorname{Rec}_{-} M$ event. This event is enabled if the first message in Channel $l_{j}$ is any $M$-type message $\left(M, f_{1}, \ldots, f_{n}\right)$. Its occurrence removes the message $\left(M, f_{1}, \ldots, f_{n}\right)$ from Channel $l_{j}$ and updates the values of variables of $\mathrm{P}_{i}$.

3. An internal event of $P_{i}$ involves no messages. It is enabled if the entity variables of $P_{i}$ satisfy a specified predicate. Its occurrence updates the values of the entity variables. Internal events are used to model interactions of the entity with its local user or channel controller, as well as timeouts and other internal transitions of the entity.

Note that both send and receive events affect the state of a channel, as well as the state of the entity.

We now describe the channel events. For $i=1$ and $i=2$, the channel loss event for channel $\mathrm{C}_{i}$ is enabled whenever Channel C $_{i}$ is not empty. Its occurrence deletes the first (message, age) pair in Channel . (Recall that the channel behavior 
in Section 2.1 requires only that the first message in each channel may be lost. There is no loss of generality here because every message must become the first message in the channel before it can be received and checked for errors.)

We now define the local time events and the global time event for the protocol model. For each local time variable $t$ in $\mathrm{P}_{i}$, there is a local time event whose occurrence ages $t$; this event is enabled if its occurrence does not cause $t$ to violate its accuracy axiom or any time axiom involving $t$. There is one global time event whose occurrence ages all global time variables, including the age values in Channel $_{1}$ and Channel Ch $_{2}$ This time event is enabled if its action does not cause any of the time or accuracy axioms to be violated, or result in an age value in Channel $_{i}$ that exceeds MaxDelay Mor $_{i}=1$ and $i=2$.

For each entity, we assume mutual exclusion between the occurrence of events of that entity. Furthermore, we assume that simultaneous occurrences of events in different components of the protocol system can be represented as an arbitrary sequence of occurrences of the same events. This latter assumption is reasonable because events in communication protocol systems can usually be defined in such a way that their occurrences are instantaneous.

\subsection{HDLC Messages}

Messages sent by $P_{1}$.

Each of the message types of $P_{1}$ has a Poll bit-field (abbreviated as $P$ field) that can take the value 0 or 1 . Any message with the $P$ field set to 1 is referred to as a Poll.

1. (U, P, Command)

This $U$ message type represents the Unnumbered frames sent by $\mathrm{P}_{1}$ for connection management. The Command field can take the value SARM or DISC. SARM stands for Set Asynchronous Response Mode, and requests $\mathrm{P}_{2}$ to go on-line. DISC stands for Disconnect, and requests $\mathrm{P}_{2}$ to go off-line.

2. (I, P, Data, NS, NR)

This I message type represents the Information frames sent by $\mathrm{P}_{1}$ for transporting data blocks to $\mathrm{P}_{2}$. Let DATABLOCKS denote the set of data blocks that can be transported by the HDLC protocol. The Data field contains a user data block, and can take any value from DATABLOCKS. NS and NR are sequence numbers that take values from $\{0,1, \ldots, N-1\}$. ( $N$ is 8 for normal HDLC operation and 128 for extended HDLC operation.) NS is referred to as the send sequence number, and is used to identify the position of the data block in the sequence of user data blocks. Successive user data blocks are sent with increasing send sequence numbers (modulo $N$ ). NR is referred to as the receive sequence number, and indicates the send sequence number of the I frame next expected at $\mathrm{P}_{1} . N R$ is an acknowledgement for data flowing in the reverse direction (i.e., from $\mathrm{P}_{2}$ to $\mathrm{P}_{1}$ ), and acknowledges all data blocks with send sequence numbers up to $N R-1$. Finally, an I frame with $P$ field set to 1 indicates that $P_{1}$ is ready to receive data from $P_{2}$.

3. (S, P, RStatus, NR)

This $\mathrm{S}$ message type represents the Supervisory frames sent by $\mathrm{P}_{1}$ for flow control and acknowledgement. The RStatus field can take the value $R R$ or RNR, indicating that $P_{1}$ is respectively Ready or Not Ready to receive data 
from $\mathrm{P}_{2}$. The $N R$ field is the receive sequence number and has been described above.

Messages sent by $P_{2}$.

Each of the message types of $\mathrm{P}_{2}$ has a Final bit-field (abbreviated as $F$ field) that can take the value 0 or 1 . Any message with the $F$ field set to 1 is referred to as a Final. $\mathrm{P}_{2}$ responds to a received Poll by sending a Final at the earliest opportunity.

1. (U, $F$, Response)

This $\mathrm{U}$ message type represents the Unnumbered frames sent by $\mathrm{P}_{2}$. The Response field can take the value UA or DM. UA stands for Unnumbered Acknowledgement, and is sent to acknowledge reception of and compliance with a $U$ command received from $P_{1}$. DM stands for Disconnected Mode, and is sent when $P_{2}$ is off-line as a response to any message (except for SARM) received from $P_{1}$.

2. (I, F, Data, NS, NR)

This I message type represents Information frames sent by $\mathrm{P}_{2}$. The Data, NS and $N R$ fields are similar to those in the $\mathrm{I}$ frames sent by $\mathrm{P}_{1}$ (except that the roles of $\mathrm{P}_{1}$ and $\mathrm{P}_{2}$ are interchanged). Also, an $\mathrm{I}$ frame with the $F$ field set to 1 indicates that $P_{2}$ is ready to receive data from $P_{1}$.

3. (S, F, RStatus, NR)

This $\mathrm{S}$ message type represents Supervisory frames sent by $\mathrm{P}_{2}$. The RStatus and $N R$ fields are similar to those in the $S$ frames sent by $\mathrm{P}_{1}$ (except that the roles of $P_{1}$ and $P_{2}$ are interchanged).

Note that message types sent by $P_{1}$ and $P_{2}$ have similar names. This should, however, cause no confusion. (The $P$ and $F$ fields actually occupy the same bit position in HDLC frames. That bit is referred to as the $P / F$ bit [7].)

\subsection{Variables of the HDLC Protocol Entities}

Variables of $P_{1}$.

$P_{1}$, the primary HDLC entity, has the following variables (the domain of each variable is also listed using a Pascal-like notation).

\{The following variables are primarily used in the Poll/Final cycle.\}

Poll_bit: $(0,1)$;

$\left\{\right.$ Poll_bit $=1$ indicates that the next message to be sent by $P_{1}$ is a Poll. Initially, Poll_bit $=0$.

Poll_Timer: (Off, 0, 1, 2, .., PollTimeoutValue);

$\{$ Poll_timer is a local time variable which is active ( $\neq$ Off) if and only if a Poll is outstanding; an active $\mathrm{Poll}_{-}$Timer indicates the time elapsed since the Poll was sent. Poll_Timer is reset to Off either upon receiving the acknowledging Final, or when Poll_Timer $=$ PollTimeoutValue (Timeout event). Initially, Poll_Timer $=$ Off. $\}$

\$Poll_ Timer: (Off, 0, 1, 2, ...);

$\left\{\$ P o l l_{-}\right.$Timer is the global time variable associated with Poll_Timer. Initially, $\$$ Poll__Timer $=$ Off. $\}$ 
Poll_Retry_Count: (0, 1, . ., MaxRetryCount);

Poll_Retry_Count indicates the number of Timeouts that have occurred since the last Final was received. Initially, Poll_Retry_Count $=0$.

\{The following variable is primarily used in connection management.\}

Mode: (Open, Opening, Closed, Closing, LinkFailure);

\{Mode indicates the status of the data link as perceived by $\mathrm{P}_{1}$. Mode is set to LinkFailure when Poll_Retry_Count exceeds MaxRetryCount. Initially, Mode $=$ Closed. $\}$

\{The following variables are primarily used in sending data blocks to $\mathrm{P}_{2}$.\}

Source: $\operatorname{array}[0 \ldots \infty]$ of $D A T A B L O C K S$;

\{Source is a history variable that records the data blocks given by the local user to $P_{1}$ to send to the remote user at $P_{2}$.

User_in, $S, A: 0 \ldots \infty$

$\left\{U_{s e r}\right.$ in, $S$ and $A$ are pointers to Source (see Figure $\left.3 a\right)$. User_in points to where the local user places his next data block. $S$ points to the data block to be next sent to $P_{2}$. $A$ points to the data block to be next acknowledged by $P_{2}$. All three pointers are intialized to 0 when the data link is opened (when Mode is set to the value Open). Data blocks from Source [A] to Source [User_in - 1] are saved in a buffer of size SBuffSize.]

$V S, V A, V C S: 0 \ldots N-1$;

$\{V S, V A$ and $V C S$ are pointers (modulo $N$ ) to Source. $V S(V A)$ indicates the send sequence number of the next data block to be sent (acknowledged). VS and $V A$ are initialized to 0 when the data link is opened. $V C S$ is described below.\}

Checkpoint_Cycle: Boolean;

\{Checkpoint_Cycle is set to True when a Poll is sent and data is outstanding; $V C S$ is set to the sequence number of the most recently sent data block. Checkpoint_Cycle is set to False when either the data block indicated by VCS is acknowledged, or a Final is received and that data block remains unacknowledged. In the latter case, retransmission of data blocks starting from VCS is initiated. Checkpoint_Cycle is initialized to False when the data link is opened.\}

Remote_RStatus: (RR, RNR);

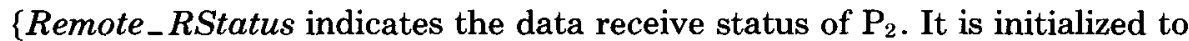
$\mathrm{RR}$ when the data link is opened.\}

\{The following variables are primarily used in receiving data blocks from $P_{2}$.

Sink: array $[0 \ldots \infty]$ of $D A T A B L O C K S$;

\{Sink is a history variable that records the sequence of data blocks received from $\mathrm{P}_{2}$ and to be delivered to the local user.\}

User_out, $R: 0 \ldots \infty$

\{User_out and $R$ are pointers to Sink (see Figure $3 \mathrm{~b}$ ). User_out points to the data block to be next delivered to the local user. $R$ points to where the 


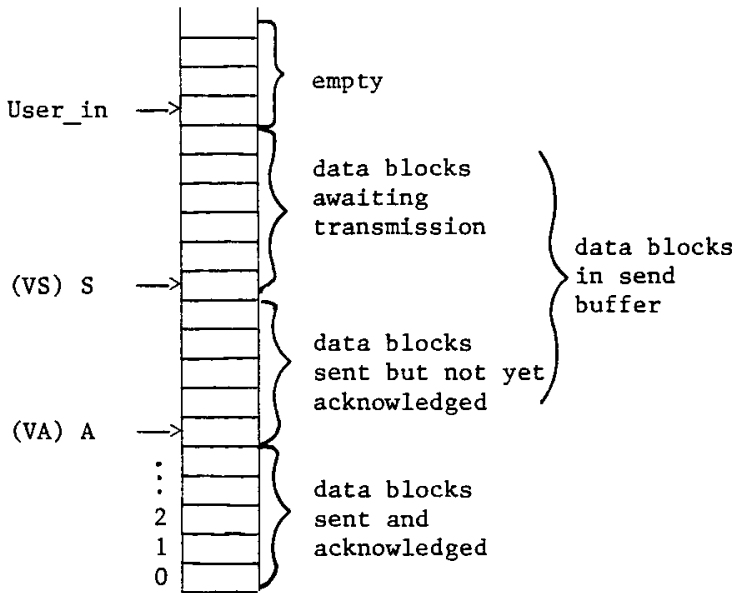

(a)

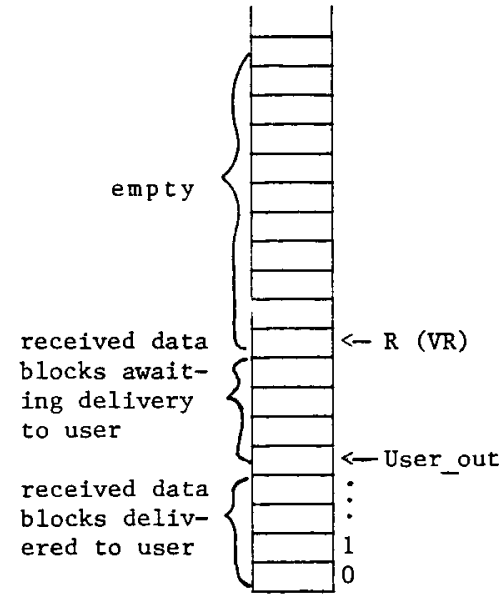

(b)

Fig. 3. Pictorial representation of pointer positions for source and sink history arrays: (a) source array; (b) sink array.

next data block received in sequence from $\mathrm{P}_{2}$ will be placed. Both pointers are initialized to 0 when the data link is opened. Data blocks from Sink [User_out] to $\operatorname{Sink}[R-1]$ are saved in a buffer of size RBuffSize.

$V R: 0 \ldots N-1$;

(VR is a pointer (modulo $N$ ) to Sink, and indicates the sequence number of the data block next expected. $V R$ is initialized to 0 when the data link is opened.\}

Local_RStatus: (RR, RNR);

$\left\{\right.$ Local_RStatus indicates the data receive status of $\mathrm{P}_{1}$. It is initialized to $\mathrm{RR}$ when the data link is opened.\}

Variables of $P_{2}$.

$\mathrm{P}_{2}$, the secondary HDLC entity, has the following variables (along with their domains):

\{The following variables are primarily used in the Poll/Final cycle.\}

Final_bit: $(0,1)$;

$\{$ Final_bit $=1$ if and only if a Poll has been received and the acknowledging Final not yet sent. Initially, Final_bit $=0$. $\}$

\$Response_Time: (Off, 0, 1, 2, .., MaxResponseTime);

$\{\$$ Response Time is an auxiliary global time variable which is active when Final_bit $=1$; when active it indicates the time elapsed since receiving the Poll. Initially, \$Response_Time = Off. $\}$

\{The following variables are primarily used in connection management.\}

Mode: (Open, Opening, Closed, Closing);

\{Mode indicates the status of the data link as perceived by $\mathbf{P}_{2}$. Initially, Mode $=$ Closed. $\}$ 
$U_{-}$Response: (UA, DM, None);

$\left\{U_{-}\right.$Response indicates the kind of $\mathrm{U}$ message to be next sent by $\mathrm{P}_{2}$. Initially, $U_{-}$Response $=$None. $\}$

\{The meaning and usage of the remaining variables are as described for $P_{1}$, except that the roles of $\mathrm{P}_{1}$ and $\mathrm{P}_{2}$ are interchanged. Also, in checkpointing, the roles of Poll and Final are interchanged.\}

\{The following variables are primarily used in sending data blocks to $P_{1}$ \}.

Source: array $[0 \ldots \infty]$ of $D A T A B L O C K S$; \{history variable of data blocks\}

User_in, $S, A: 0 \ldots \infty$;

$V S, V A, V C S: 0 \ldots N-1$;

Checkpoint_Cycle: Boolean;

\{pointers to Source\}

\{pointer variables modulo $N$ \}

Remote_RStatus: (RR, RNR);

\{The following variables are primarily used in receiving data blocks from $\mathrm{P}_{1}$ \}.

Sink: array[0 . $\infty]$ of DATABLOCKS; \{history variable of data blocks\}

User_out, $R: 0 \ldots \infty$;

$V R: 0 \ldots N-1$;

Local RStatus: (RR, RNR);

(pointers to Sink\}

\{pointer variable modulo $N$ \}

Note that many variables in $P_{1}$ and $P_{2}$ have the same names. Wherever this might cause ambiguity, we qualify the variable names with numerical subscripts, for instance, Mode ${ }_{1}$ and Mode . $_{2}$

\subsection{Events of the HDLC Protocol}

The events of the HDLC protocol system are formally specified in Tables I-V (all tables may be found in Appendix B). (A prose description can be found in [19].) The events of the entities are shown in Tables I and II. The program statements in upper case (POLL_SENT, FINAL_RECEIVED, INITIALIZE_ SEND_VARIABLES, etc.) stand for code segments that are shown in Table III. When used in an entity event, the variables they refer to are the variables of that entity. We use the notation $\oplus$ and $\theta$ to refer to addition modulo $N$ and subtraction modulo $N$ respectively. Poll_Timer (in $\mathrm{P}_{1}$ ) has the accuracy axiom $\mid$ Poll_Timer-\$Poll_Timer $\mid \leq 1+a\left(\$ P_{\text {Poll_Timer }}\right)$, where $a$ is the maximum relative error in Poll_Timer's clock frequency. $\mathrm{P}_{2}$ satisfies the local time axiom $\$$ Response_Time $\leq$ MaxResponseTime. Finally, in order to have at most one Poll outstanding at any time, we assume that PollTimeoutValue $>1+(1+$ a) $\left(\right.$ MaxDelay $_{1}+$ MaxResponseTime + MaxDelay $\left._{2}\right)$.

The channel events are specified in Table IV.

The time events of the HDLC protocol are specified in Table V. Poll_Timer_ Tick is the local time event for Poll__Timer. Global_Tick is the global time event of the system. The procedure Age (in the actions of the time events) ages all its time variable arguments by one tick. Note that the global time event cannot age \$Response _Time beyond MaxResponseTime, nor can it cause a message to stay in Channel Cor longer than MaxDelay $_{i}$, nor can it cause Poll_Timer to be more inaccurate than as specified by its accuracy axiom. Similarly Poll_Timer_Tick 
cannot cause Poll_ Timer to be more inaccurate than as specified by its accuracy axiom.

The initial state of the HDLC protocol system is given by the following value assignments to the protocol system variables: both Channel $l_{1}$ and Channel $_{2}$ are empty; entity variables have the initial values specified in Section 2.4. Note that some of the entity variables concerned primarily with data transfer functions are not initialized until the data link is opened (Mode set to the value of Open).

\section{IMAGE PROTOCOLS AND SAFETY PROPERTIES}

The HDLC protocol described offers three distinguishable functions to the users: connection management, and one-way data transfers in two directions. We would like to examine the logical behavior of the HDLC protocol with respect to these functions.

We note that these three functions are not independent of each other. Dependencies of the two types described in Section 1.1 (shared variables and shared protocol messages) are present. We mention some of them here. First, the Poll/ Final cycle (a handshaking mechanism in the protocol) is used by all three functions; that is, Poll and Final messages, Poll_bit, Poll_Timer, Poll_Retry_ Count and Final_bit are shared by all three functions. Second, the connection management function interacts with the data transfer functions at opening (when the data transfer variables are initialized), and at closing (when data transfer is inhibited); the variable Mode in each entity, and the SARM, DISC and UA messages are shared by all three functions. Third, the data transfers in the two directions interact through I frames that carry data in one direction and acknowledgment for data in the opposite direction. In addition, an incoming I frame with the Poll (Final) field set to 1 conveys flow control information for outgoing data.

Such dependencies present major obstacles for protocol analysis using a decomposition approach. We use the approach of protocol projections to obtain an image protocol for each function. In Section 3.1, we outline a procedure for constructing image protocols of sufficient resolution to verify desired logical properties of individual functions. We also briefly describe how to obtain inductively complete assertions that imply desired safety properties.

An assertion is inductively complete for a protocol system if (a) the initial condition of the protocol system satisfies the assertion, and (b) for each event in the protocol, given that the assertion holds before the event occurrence, the enabling condition and the action of the event are sufficient to show that the assertion holds after the event occurrence. In Sections 3.2, 3.3 and 3.4, we use the approach outlined in Section 3.1 to obtain image protocols for each of the HDLC functions, and state inductively complete assertions that imply some desired safety properties.

In Section 3.5, we describe a minor modification to HDLC that allows us to obtain small well-formed image protocols for the three functions of interest. The modification involves the addition of a one-bit flow control field to the HDLC information frames. These well-formed image protocols are faithful to the HDLC protocol. (We have not, however, investigated liveness properties of the HDLC protocol or of our modified version of the HDLC protocol in this paper.) We 
propose that this modified HDLC protocol can be considered to be well-structured.

\subsection{Verification Via Projections}

In this section, we briefly outline the method of protocol projections. In Section 3.1.1, we define image protocols. In Section 3.1.2, we describe two iterative methods for generating an image protocol of sufficient resolution to verify a specified property $A_{0}$ of a given function. For a detailed and formal presentation of the theory and methodology, see $[13,16,20]$.

3.1.1. Image Protocol Definition. An image protocol is defined with respect to a given subset of entity variables of the original protocol. For $i=1$ and $i=2$, let $V_{i}$ denote the set of entity variables of $P_{i}$ in the HDLC protocol, and let $V_{i}^{\prime}$ denote the subset of $V_{i}$ representing the entity variables of an image protocol. Messages and events of the image protocol are defined based on $V_{1}^{\prime}$ and $V_{2}^{\prime}$.

Each value assignment to the variables in $V_{i}$ represents a state $s$ of entity $P_{i}$. The portion of this value assignment that corresponds to variables in $V_{i}^{\prime}$ represents the image $s^{\prime}$ of $s$.

For each message type $(M, \underline{F})$ sent by $\mathrm{P}_{i}$ (where $\underline{F}$ denotes the fields of $M$ ), its image is denoted by $\left(M^{\prime}, \underline{F}^{\prime}\right)$, where $\underline{F}^{\prime}$ is obtained by deleting those fields in $\underline{E}$ that do not affect variables in $\mathrm{V}_{j}^{\prime}(j \neq i)$ in the $\operatorname{Rec}_{-} M$ event of $\mathrm{P}_{j}$. The image of any $M$-type message $(M, f)$ is given by $\left(M^{\prime}, \underline{f}^{\prime}\right)$, where $\underline{f}^{\prime}$ consists of those field values in $f$ corresponding to $\underline{F}^{\prime}$.

Each HDLC entity event specifies a set of entity state transitions (which involve messages in the case of send and receive events). For example, the Send $-M$ event, which involves variables in $\mathrm{V}_{i}$ and fields in $\underline{E}$, specifies transitions of the form $(s, r,(M, f))$; that is, when $\mathrm{P}_{i}$ is in some state $s$ satisfying the enabling condition of Send $-\bar{M}$, the action of Send_M leaves $\mathrm{P}_{i}$ in some state $r$ and sends message $(M, f)$. The image of Send $M_{-}$is an event Send $M_{-}^{\prime}$ involving $\mathrm{V}_{i}^{\prime}$ and $E^{\prime}$, and satisfying the following: Send_ $M^{\prime}$ specifies a transition $\left(s^{\prime}, r^{\prime},\left(M^{\prime}, f^{\prime}\right)\right)$ if and only if Send $-M$ specifies a transition $(s, r,(M, f))$ for some $s, r$ and $(M, \underline{f})$ whose images are $s^{\prime}, r^{\prime}$ and $\left(M^{\prime}, f^{\prime}\right)$ respectively. The images of receive events and internal events are similarly defined. (The image events were obtained directly from the HDLC event descriptions without explicitly considering their state transitions [16].)

The image message types and the image entity events serve as the message types and entity events of the image protocol. Also, image message types and image entity events that have no effect on variables in $V_{1}^{\prime}$ and $V_{2}^{\prime}$ are eliminated in the image protocol; those that have identical effects are merged. The behavior of the communication channels is the same in the image protocol system as in the HDLC protocol system. The initial values of the image protocol variables are the same as the initial values of the corresponding HDLC variables.

An image protocol as constructed above captures only part of the behavior of the original protocol. However, we have shown that a safety property that holds in the image protocol also holds in the original protocol. Also, well-formed image protocols are faithful to the original protocol in all of their safety and liveness properties. Informally, an image protocol is well-formed if the following holds: in 
the image protocol, if an entity event $e^{\prime}$ takes the entity from state $r^{\prime}$ to state $s^{\prime}$ and involves message $m^{\prime}$, then in the original protocol, from every entity state $r$ whose image is $r^{\prime}$ there is a sequence of entity events that will take the original protocol to a state where an event $e$ whose image is $e^{\prime}$ can occur $[13,16]$.

We have not investigated liveness properties of the HDLC protocol. We can decide whether certain states will be eventually reached (a typical liveness assertion) by examining the execution paths of the system. The expressive power of our model for such liveness assertions is limited, when compared with models that incorporate temporal logic semantics [15]. Recall however that communication protocols are time-dependent systems, and that progress properties of such systems can be stated as safety assertions involving time variables.

3.1.2. Constructing an Image Protocol of Desired Resolution. We next describe an iterative method that attempts to find the smallest image protocol that is of sufficient resolution to verify a desired safety property $A_{0}$ of the projected function.

For $i=1$ and $i=2$, let $\mathrm{V}_{i}^{\prime}$ denote the entity variables of $\mathrm{P}_{i}$ that appear in the assertion $\mathrm{A}_{0}$. We first describe the iterative step. Construct an image protocol using $V_{1}^{\prime}$ and $V_{2}^{\prime}$ as the set of entity variables. Verify if assertion $A_{0}$ holds in this image protocol (see below). If it does, then from the properties of image protocols $\mathrm{A}_{0}$ holds in the HDLC protocol and the verification is over. If $\mathrm{A}_{0}$ does not hold in the image protocol, then there is a sequence of events, referred to as a test sequence, that takes the image protocol from its initial condition to global state that violates $\mathrm{A}_{0}$ (see below). Consider the event sequences in the HDLC protocol that have images equal to the test sequence. If any of these HDLC event sequences can occur in the HDLC protocol, then $A_{0}$ does not hold in the HDLC protocol, and the verification is over. If none of these HDLC event sequences can occur, that is because some HDLC variables (not included in $V_{1}^{\prime} \cup V_{2}^{\prime}$ ) inhibit certain events from occurring. Include these variables in $V_{i}^{\prime}$ and repeat the above iterative step until termination. In the worst case, termination occurs with the image protocol being equal to the original protocol.

Although this approach may at first appear to be inefficient, since in each iteration we check whether $A_{0}$ holds for an image protocol, this is in fact not the case because at each iteration, even if we cannot verify $A_{0}$, we can usually establish properties of the image protocol that are helpful in verifying $A_{0}$. (For example, the image protocol may not transfer data correctly, but it does ensure correct Poll/Final handshake and data link initialization.) Since each succeeding image protocol is a refinement of all earlier image protocols, these properties, once established, need not be verified again.

To verify $A_{0}$ for an image protocol, we do an iterative search for an inductively complete safety assertion $A$ that implies $A_{0}$. Initially $A$ equals $A_{0}$. The iterative step is as follows. For each event $e$ of the image protocol, determine the weakest precondition [5] that must hold before the occurrence of $e$ in order that A holds immediately after the occurrence of $e$. Let $\mathrm{C}$ denote the conjunction of $\mathrm{A}$ and all the weakest preconditions. If $\mathrm{C}$ is equivalent to $\mathrm{A}$ and the initial state of the image protocol satisfies $C$, then $A$ is inductively complete, $A_{0}$ holds for the image protocol, and the search is over. If the initial state of the image protocol does not satisfy $\mathrm{C}$, then by examining the trace of the iterations, a test sequence can be 
determined that takes the image protocol from its initial state to a state violating $A_{0}$. In this case, $A_{0}$ does not hold and the search is over.

If neither of the above conditions holds, then replace $A$ by $C$ and repeat the iteration. If at any point we determine (by inspection) that a safety property $T$ holds for the image protocol, then $A$ can be replaced by the conjunction of $A$ and $\mathrm{T}$. This speeds up the search by constraining the weakest preconditions.

The above iterative method can be used only for verifying safety assertions. A variation on this method can be used when $A_{0}$ is either a safety or liveness assertion. In this second method, each image protocol that is obtained is tested for well-formedness (instead of testing whether $\mathrm{A}_{0}$ holds). If the test for wellformedness fails, then the failure will point out additional HDLC entity variables to include in constructing the next image protocol. Repeat this step until a wellformed image protocol is obtained. Since well-formed image protocols are faithful, any outcome which results from verifying $A_{0}$ on the image protocol is valid for the original protocol.

Note that the check for well-formedness involves an examination of each protocol entity individually. It does not involve an analysis of the global interaction of the intermediate image protocols. Hence, this checking can be performed efficiently. On the other hand, given a safety assertion $A_{0}$, the image protocol resulting from the first method is usually smaller (never larger) than that obtained from the second method. Since real-life communication protocols are typically time-dependent systems with real-time specifications stated as safety properties, the first method is usually more useful in practice.

\subsection{Image Protocol for Connection Management}

The first image protocol we show is for the connection management function. A desirable property of the HDLC protocol with respect to this function may be stated as follows:

$$
\left(\text { Mode }_{1}=\text { Open } \Rightarrow \text { Mode }_{2}=\text { Open }\right) \text { and }\left(M_{\text {ode }}=\text { Closed } \Rightarrow \text { Mode } e_{2}=\text { Closed }\right)
$$

This property can be stated using only the variables Mode in $\mathrm{P}_{1}$ and Mode in $P_{2}$. Starting from this initial set of entity variables, and applying the first method, we determine that the following variables are also needed: Poll_bit, Poll_Timer, $\$ P o l l_{-}$Timer and Poll_Retry_Count in $\mathrm{P}_{1}$; Final_bit, \$Response_Time and $U_{-}$Response in $\mathrm{P}_{2}$. The resulting image protocol (described below) is also wellformed-using the second method would result in the same image protocol.

The images of the HDLC message types can now be defined as follows. First, there are the message types sent by $P_{1}$. The image of message type (U, $P$, Command) is defined by (U', $P$, Command) (i.e., all the fields of the U Message type are needed in the image protocol). The image of both (I, P, Data, NS, NR) and $(\mathrm{S}, P, R$ Status, $N R)$ can be defined by $\left(\mathrm{I}^{\prime} P\right)$ where $\mathrm{I}^{\prime}$ denotes a (new) message type that corresponds to either an I or an S frame of the HDLC protocol. Next, there are the message types sent by $\mathrm{P}_{2}$. The image of message type (U, $F$, Response) is ( $\mathrm{U}^{\prime}, F$, Response). The image of both (I, F, Data, NS, NR) and (S, $F, R$ Status, $N R)$ can be defined by $\left(\mathrm{I}^{\prime}, F\right)$. Thus $\left(\mathrm{U}^{\prime}, P\right.$, Command) and $\left(\mathrm{I}^{\prime}, P\right)$ are the message types sent by $\mathrm{P}_{1}$, and $\left(\mathrm{U}^{\prime}, F\right.$, response) and $\left(\mathrm{I}^{\prime}, F\right)$ are the message types sent by $P_{2}$. 
The entity events of the image protocol are displayed in Tables VI and VII. The channel and time events of the image protocol are exactly as the channel and time events shown in Tables IV and V for the HDLC protocol. These events are obtained by taking the images of the corresponding events of the HDLC protocol (Tables I, II, IV and V). In the event Send_U' of Table VI, the enabling condition and action are the same as in the event Send_U of Table I, except that $\mathrm{U}$ is replaced by $\mathrm{U}^{\prime}$. This notation is also used in the remaining tables.

The initial state of this image protocol is obtained from the HDLC protocol: Mode $_{1}=$ Mode $_{2}=$ Closed, $U_{-}$Response $=$None, Poll $\_$Timer $=\$$ Poll - Timer $=$

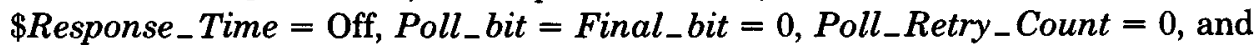
Channel $_{1}$ and Channel Che $_{2}$ are empty.

The reader is referred to [16] for additional details.

3.2.1. Safety properties. For this image protocol, the following assertion concerning the Poll/Final cycle has been shown to be inductively complete, hence invariant. (A proof can be found in Appendix A. It is shown as an illustration of our technique. Proofs of other assertions to be introduced below are omitted for brevity.) In the assertions, $\$ A g e$ denotes the age of the associated message in the channel.

Poll/Final (PF) Assertions.

PF1. Poll_bit $=1 \Rightarrow$ Poll_$_{-}$Timer $=$Off

PF2. Poll_ Timer $=$ Off $\Rightarrow$ No Poll in Channel 1

and Final_bit $=0_{0}$

and no Final in Channel $_{2}$

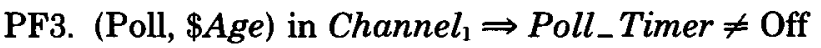

and $\$ P$ Poll_timer $=\$ A g e$

and exactly one Poll in Channel

and Final_bit $=0$

and no Final in Channel $_{2}$

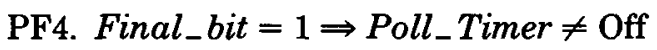

and $\$$ Poll_Timer $\leq$ MaxDelay 1 + $\$$ Response_Time

and no Poll in Channel

and no Final in Channel

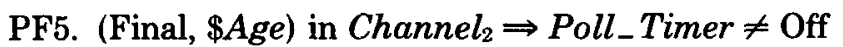

and $\$$ Poll_Timer $\leq$ MaxDelay ${ }_{1}+$ MaxResponseTime $+\$ A g e$

and no Poll in Channel

and Final_bit $=0$

and exactly one Final in Channel

For this image protocol, the conjunction of the PF assertions and the following assertions concerning connection management are inductively complete, hence invariant (proof in $[16,18]$ ).

Connection Management (CM) Assertions.

CM1. (a) Mode $_{1}=$ Open $\Rightarrow$ Mode $_{2}=$ Open and no U' frames in Channel 1 and no $\mathrm{U}^{\prime}$ frames in Channel $l_{2}$ and $U_{-}$Response $=$None 
(b) Mode $_{1}=$ Closed $\Rightarrow$ Mode $_{2}=$ Closed and Channel C $_{1}$ is empty and Channel $_{2}$ is empty and $U_{-}$Response $=$None

(c) $\left(\mathrm{I}^{\prime}, P\right)$ in Channel $_{1} \Rightarrow$ Mode $_{2}=$ Open

CM2. Poll_ Timer $=$ Off $\Rightarrow$ no $U^{\prime}$ frames in Channel ${ }_{1}$ and no $\mathrm{U}^{\prime}$ frames in Channel $\mathrm{C}_{2}$ and $U_{-}$Response $=$None and $\left(M_{o d e}=\right.$ Open or $M o d e_{2}=$ Closed)

CM3. (U', $P$, Command) in Channel $\Rightarrow$ exactly one $\mathrm{U}^{\prime}$ frame in Channel Ch $_{1}$ and $\left(\mathrm{U}^{\prime}, 1\right.$, Command) is at tail of Channel ${ }_{1}$ and $\left(\right.$ Command $=\mathrm{SARM} \Rightarrow$ Mode $_{1}=$ Opening) and (Command $=$ DISC $\Rightarrow$ Mode $_{1}=$ Closing) and $\left(\right.$ Mode $_{2}=$ Open or Mode $_{2}=$ Closed) and $U_{-}$Response $=$None and no $\mathrm{U}^{\prime}$ frames in Channel $\mathrm{C}_{2}$

CM4. $U_{-}$Response $\neq$None $\Rightarrow$ Final__bit $=1$ and Channel C $_{1}$ is empty and Channel ${ }_{2}$ has no $\mathrm{U}^{\prime}$ frames and $\left(U_{-}\right.$Response $=\mathrm{UA} \Rightarrow\left(\right.$ Mode $_{1}=$ Mode $_{2}=$ Opening or Mode $_{1}=$ Mode $_{2}=$ Closing)) and $\left(U_{-}\right.$Response $=\mathrm{DM} \Rightarrow\left(\right.$ Mode $_{1}=$ Closing and Mode $_{2}=$ Closed))

CM5. (U', $F$, Response) in Channel $\Rightarrow$ Channel $_{1}$ empty and exactly one $U^{\prime}$ frame in Channel $_{2}$ and $F=1$ and (Response $=\mathrm{DM} \Rightarrow$ Mode $_{2}=$ Closed and Mode $_{1}=$ Closing) and (Response $=\mathrm{UA} \Rightarrow\left(\right.$ Mode $_{2}=$ Closed and Mode $_{1}=$ Closing) or $\left(\right.$ Mode $_{2}=$ Open and Mode $_{1}=$ Opening)) and $U_{-}$Response $=$None

\subsection{Image Protocol for $P_{1}$ to $P_{2}$ Data Transfer}

We now consider the function of one-way data transfer from $P_{1}$ to $P_{2}$. Two desirable properties of the HDLC protocol with respect to this function may be stated as follows.

If Mode $_{1}=$ Mode $_{2}=$ Open then

1. $\operatorname{Sink}_{2}[i]=$ Source $_{1}[i]$ for $0 \leq i<$ User_out $_{2}$

2. $0 \leq A_{1} \leq S_{1}<A_{1}+N$

The first property states that data is transferred in sequence; the second that the maximum number of outstanding data blocks (and therefore the minimum storage requirement) at $P_{1}$ is $N-1$. These properties can be stated using only the variables Mode, Source, $A$ and $S$ in $\mathrm{P}_{1}$, and Mode, Sink and User_out at $\mathrm{P}_{2}$.

Starting from this initial set of entity variables, and applying the first method, we determine that the following variables are needed: Poll_bit, Poll_Timer, $\$ P o l l_{-}$Timer, Poll_Retry_Count, User_in, VS, VA, VCS, Checkpoint_Cycle and

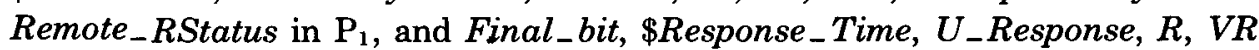
and Local_RStatus in $\mathrm{P}_{2}$. The resulting image protocol (described below) has sufficient resolution to verify the desired safety property. However, it is not wellformed. In fact, we have shown in [16] that to obtain a well-formed image protocol for this function, we would have to include almost the entire HDLC protocol. 
We now define the images of the HDLC message types sent by $P_{1}$. The image of message type (U, $P$, Command) is $\left(\mathrm{U}^{\prime}, P\right.$, Command). The image of (I, $P$, Data, NS, NR) is defined as $\left(\mathrm{I}^{\prime}, P, D a t a, N S\right)$, where $\mathrm{I}^{\prime}$ is a new message type that corresponds to an I frame of the HDLC protocol. The image of $(\mathrm{S}, P$, RStatus, $N R$ ) is $\left(\mathrm{S}^{\prime}, P\right)$, where $\mathrm{S}^{\prime}$ is a new message type corresponding to an $\mathrm{S}$ frame of the HDLC protocol.

Next, we define the images of the HDLC message types sent by $\mathrm{P}_{2}$. The image of (U, $F$, Response) is ( $\mathrm{U}^{\prime}, F$, Response). The image of (I, $F$, Data, NS, NR) is $\left(\mathrm{I}^{\prime}, F, N R\right)$, where $\mathrm{I}^{\prime}$ denotes a new message type corresponding to an $\mathrm{I}$ frame of the HDLC protocol. The image of (S, F, RStatus, $N R$ ) is $\left(\mathrm{S}^{\prime}, F\right.$, RStatus, NR) .

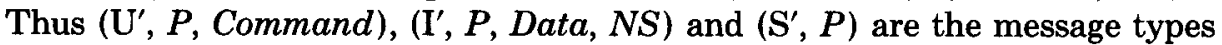
sent by $\mathrm{P}_{1}$, and $\left(\mathrm{U}^{\prime}, F\right.$, Response $),\left(\mathrm{I}^{\prime}, F, N R\right)$ and $\left(\mathrm{S}^{\prime}, F, R\right.$ Status, $\left.N R\right)$ are the message types sent by $\mathrm{P}_{2}$.

The events of the image protocol system are obtained by taking the images of the HDLC protocol system [16]. The events of the protocol entities in the image protocol are shown in Tables VIII and IX. The channel and time events for this protocol are exactly as described in Tables IV and V. The initial state of this protocol is the same as that of the HDLC protocol.

We note that the image protocol does in fact display a minor liveness property that the original protocol does not have: the number of $\mathrm{I}^{\prime}$ frames in channel $\mathrm{C}_{2}$ can exceed $N$. In fact, the Send_I' event of $\mathrm{P}_{2}$ is the only event that causes the image protocol not to be well-formed [16]. In Section 3.5, we suggest a minor modification to HDLC that will correct this and allow us to construct small wellformed image protocols for the one-way data transfer functions, as well as for the connection management function.

3.3.1. Safety Properties. For this image protocol, we have verified (by using the method described in Section 3.1.2) the desired safety properties of the projected function.

\section{Notation.}

We first describe some notation that is used in the assertion. Recall that Channel $l_{1}$ and $C h a n n e l_{2}$ represent the sequence of messages in $\mathrm{C}_{1}$ and $\mathrm{C}_{2}$ respectively. We shall think of Channel ${ }_{1}\left(\right.$ Channel $\left._{2}\right)$ as a sequence of messages from left to right; the tail of Channel ${ }_{1}\left(\right.$ Channel $\left._{2}\right)$ is at the left, and the head of Channel $\left(\right.$ Channel $\left._{2}\right)$ is at the right. When Channel $_{1}\left(\right.$ Channel $\left._{2}\right)$ contains only one message, the tail and the head point to the same message.

The following notation is used in describing the state of Channel . Given integers $s$ and $v s$ such that $s \geq 0$ and $0 \leq v s<N$, the notation $\langle s, v s\rangle$ denotes the tuple ( $\mathrm{I}^{\prime}$, Data, NS), where Data contains Source $[s]$ and $N S$ contains $v s$. Let $x$ be a sequence whose elements are either $\mathrm{U}^{\prime}$ frames or entries of the form $\langle s, v s\rangle$. Channel $l_{1}$ is said to satisfy $x$, if, by deleting all $S^{\prime}$ frames in Channel $_{1}$ and by deleting the $P$ field in all $\mathrm{I}^{\prime}$ frames in Channel $_{1}$, the resulting sequence equals $x$. Given two consecutive elements $x_{1}, x_{2}$ in $\underline{x}$, we say that a Poll is to the immediate left of $x_{2}$ to mean that it is in between $x_{2}$ and $x_{1}$. We say that a Poll is to the left of $x_{2}$ to mean that it is anywhere to the left of $x_{2}$.

Given integers $n, s$ and $v s$ such that $0 \leq n \leq s$ and $0 \leq v s<N$, the notation $\langle s-1$, vs $\Theta 1\rangle$.. $(s-n$, vs $\Theta n\rangle$ denotes the sequence $\langle s-1$, vs $\Theta 1\rangle,\langle s-2$, 
vs $\ominus 2\rangle, \ldots,\langle s-n$, vs $\ominus n\rangle$ if $n>0$, and the empty sequence if $n=0$. Either way, $n$ is the length of the sequence. (We use a simple comma to denote concatenation.)

Finally, whenever the term [Old_Info_Sequence] appears in the assertions, it denotes any sequence (possibly empty) of entries of the form $\langle s, v s\rangle$. We use [Old_Info_Sequence] to refer to the $\langle s, v s\rangle$ sequence obtained from the I' frames in Channel $l_{1}$ pertaining to a data connection that is in the process of being reset.

The following notation is used in describing the state of Channel Chiven an $^{2}$ integer $v r$ such that $0 \leq v r<N$, the notation $[v r]$ denotes either an empty sequence or any sequence of one or more receive sequence numbers, each equalling $v r$. Let $y$ be a sequence whose elements are either $U^{\prime}$ frames or entries of the form [vr]. An instance of $y$ is any sequence of $\mathrm{U}^{\prime}$ frames and $N R$ fields obtained by (arbitrarily) fixing the length of each [vr] in $y$. Channel Ch said to $_{2}$ satisfy $y$ if, by replacing all $S^{\prime}$ and $I^{\prime}$ frames in Channel $l_{2}$ by their $N R$ fields, the resulting sequence equals an instance of $y$.

Given integers $m$ and $v r$ such that $m \geq 0$ and $0 \leq v r<N$, the notation $[v r] \ldots[v r \ominus m]$ denotes the sequence [vr], [vr $\ominus 1], \ldots,[v r \ominus m]$ if $m>0$, and the sequence [vr] if $m=0$. For convenience we assume that if $m>0$, then $[v r \ominus m]$ is not empty.

Whenever the term [Old_Ack_Sequence] occurs in the assertions, it denotes any sequence (possibly empty) of entries of the form [vr]. We use [Old_Ack Sequence] to refer to the [vr] sequence obtained from the $I^{\prime}$ and $S^{\prime}$ frames in Channel $_{2}$ pertaining to a data connection that is in the process of being reset.

Lastly, (Final, $N R$ ) denotes an $I^{\prime}$ or $S^{\prime}$ frame whose $F$ field equals 1 and whose receive sequence number equals $N R$.

\section{Data Transfer Assertions.}

The assertions A1-A6 listed below, in conjunction with the PF assertions, are inductively complete, and hence invariant (proof appears in [16, 18]. A1-A5 are concerned with conditions that hold during opening/closing of the data link; A6 is concerned with conditions of data transfer that hold when the data link is open.

Al. (a) Mode $_{1}=$ Open $\Rightarrow$ Mode $_{2}=$ Open and no U' frames in Channel and no $\mathrm{U}^{\prime}$ frames in Channel $l_{2}$ and $U_{-}$Response = None

(b) Mode $_{1}=$ Closed $\Rightarrow$ Mode $_{2}=$ Closed and Channel Ch $_{1}$ is empty and Channel $_{2}$ is empty and $U_{-}$Response = None

(c) $\left(\mathrm{I}^{\prime}, P\right.$, Data, NS) or $\left(\mathrm{S}^{\prime}, P\right)$ in Channel $_{1} \Rightarrow$ Mode $_{2}=$ Open.

A2. Poll_timer $=$ Off $\Rightarrow U_{-}$Response $=$None and $\left(\right.$ Mode $_{2}=$ Open or Mode $_{2}=$ Closed $)$.

A3. (U', $P$, Command) in Channel C $_{1}$

$\Rightarrow$ Channel $_{1}$ satisfies (U', 1, Command), [Old_Info_Sequence] and (Command $=\mathrm{SARM} \Longrightarrow$ Mode $_{1}=$ Opening)

and (Command $=$ DISC $\Rightarrow$ Mode $_{1}=$ Closing) and $\left(\right.$ Mode $_{2}=$ Open or Mode $_{2}=$ Closed) and $U_{-}$Response $=$None and Channel $_{2}$ satisfies [Old_Ack_Sequence].

A4. $U_{-}$Response $\neq$None $\Rightarrow$ Final_bit $=1$ and Channel $l_{1}$ is empty and Channel $l_{2}$ satisfies [Old_Ack_Sequence] 


$$
\begin{aligned}
& \text { and }\left(U_{-} \text {Response }=\mathrm{UA} \Rightarrow\left(\text { Mode }_{1}=\text { Mode }_{2}=\right.\text { Opening }\right. \\
& \text { or } \left.\left.\text { Mode }_{1}=\text { Mode }_{2}=\mathrm{Closing}\right)\right) \\
& \text { and }\left(U_{-} \text {Response }_{2}=\mathrm{DM} \Rightarrow\left(\text { Mode }_{1}=\right.\text { Closing }\right. \\
& \text { and } \left.\left.\text { Mode }_{2}=\text { Closed }\right)\right) .
\end{aligned}
$$

A5. (U', F, Response) in Channel $\Rightarrow$ Channel $_{1}$ empty

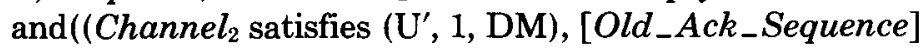
and Mode $_{2}=$ Closed and Mode $1=$ Closing) or (Channel S $_{2}$ satisfies (U', 1, UA), [Old_Ack_Sequence] and Mode $_{2}=$ Closed and Mode I $_{1}=$ Closing)

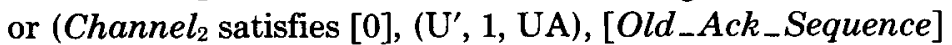
and $M o d e_{2}=$ Open, $V R=R=U s e r_{-} o u t=0, U_{-}$Response $=$None and Mode $_{1}=$ Opening)).

(A5 supplies the initial condition for the next assertion, A6, to hold when the data link is opened at $P_{1}$.

A6. If Mode $_{1}=$ Open then (Mode $e_{2}=$ Open and B1 and B2 and B3 and B4 and (B5 or B6)) holds, where B1-B6 are the assertions listed below.

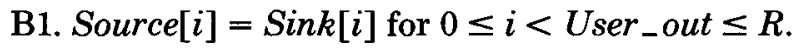

\{Data is transferred in sequence.\}

B2. $A \leq R \leq S<A+N$.

\{The number of outstanding blocks in $\mathrm{P}_{1}$ is always less than $N$.\}

B3. $A \bmod N=V A, S \bmod N=V S, R \bmod N=V R$.

\{With $\mathrm{B} 2$ above, this asserts that the modulo $N$ state variables point to the same data blocks in Source and $\operatorname{Sink}$, as pointed to by $A, S$ and $R$.

B4. Checkpoint_Cycle $=$ True $\Rightarrow V S \ominus V A>V C S \ominus V A$.

(The data blocks with sequence numbers $V A, V A \oplus 1, \ldots, V S \ominus 1$ are outstanding. When a checkpoint cycle is ongoing, then of these data blocks, the subset with sequence numbers $V A, V A \oplus 1, \ldots, V C S$ were outstanding when the Poll was sent.\}

B5. (a) Channel $l_{1}$ satisfies $(S-1, V S \ominus 1\rangle \ldots\langle S-n, V S \ominus n\rangle$ where $0 \leq n \leq$ $S-R$.

\{Channel $l_{1}$ has a (possibly empty) sequence of $\mathrm{I}^{\prime}$ frames containing successive data blocks. If $n=S-R$ then $\langle S-n, V S \ominus n\rangle$ is the next data block expected by $\mathrm{P}_{2}$. If $n<S-R$, then the data block next expected by $\mathrm{P}_{2}$ has been lost, none of the $I^{\prime}$ frames currently in Channel $_{1}$ will be accepted by $\mathrm{P}_{2}$, and $P_{1}$ is not yet aware of the loss.

(b) Channel $l_{2}$ satisfies [VR . [ [VR $\left.\ominus m\right]$ where $0 \leq m \leq R-A$.

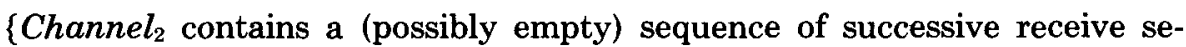
quence numbers. If $m>0$ then [VR $\ominus m$ ] consists of at least one $I^{\prime}$ or $S^{\prime}$ frame with its receive sequence number equal to $V R \ominus m$.

(c) Checkpoint_Cycle and Poll in Channel $_{1} \Rightarrow(n=0$ and VCS $=V S \ominus 1)$ or ( $n>0$ and Poll is with or to immediate left of $\langle S-i, V S \ominus i\rangle$ where $i=V S \ominus V C S$ ) 
or $(n>0$ and Poll is to right of $\langle S-n, V S \ominus n\rangle$ and $n=(V S \ominus V C S)-1)$.

\{Relates position of a checkpoint Poll in Channel $_{1}$ to VCS.\}

(d) Checkpoint_Cycle and Final_bit $=1$ and $(V R \ominus V A \leq V C S \ominus V A) \Rightarrow$ $S-n>R$.

\{If checkpoint Poll has reached $P_{2}$ and all data up to the checkpoint has not been received at $P_{2}$, then data blocks are lost.\}

(e) Checkpoint_Cycle and (Final, NR) in Channel and $(N R \ominus N A \leq V C S \ominus V A) \Rightarrow N R=V R$ and $S-n>R$.

\{If checkpoint Final is in $\mathrm{C}_{2}$ and its $N R$ does not acknowledge all data blocks up to VCS, then data blocks are lost and the Final's $N R$ equals $V R$.

B6. $\left\{P_{1}\right.$ realized (some time ago) that $P_{2}$ was receiving out of sequence $I^{\prime}$ frames, and has commenced (or is about to commence) retransmission of in sequence I' frames.\}

(a) Channel $l_{1}$ satisfies $\langle S-1, V S \Theta 1\rangle \ldots\langle S-j, V S \Theta j\rangle,\left\langle s_{0}-1, v s_{0} \Theta 1\right\rangle$ .. $\left\langle s_{0}-k, v s_{0} \ominus k\right\rangle$ where $j=S-A, A+N>s_{0}>s_{0}-k>R$, and $v s_{0}$ $=s_{0} \bmod N$.

\{The head of Channel C $_{1}$ consists of one or more old out-of-sequence I' frames $\left\langle s_{0}-1, v s_{0} \ominus 1\right\rangle \ldots\left\langle s_{0}-k_{s} v s_{0} \ominus k\right\rangle$, followed by zero or more in-sequence I' frames $(S-1, V S \ominus 1\rangle \ldots\langle S-j, V S \ominus j\rangle$.

(b) Channel 2 satisfies [VR].

\{Channel ${ }_{2}$ has zero or more receive sequence numbers, all equal to $V R$.\}

(c) $R=A$.

$\left\{\mathrm{P}_{1}\right.$ has determined the current value of $R$ exactly. Hence $\langle S-j, V S \ominus j\rangle$, the first of the new $I^{\prime}$ frames in Channel $_{1}$, is the one next expected by $\mathrm{P}_{2}$.

(d) (There is no Poll in Channel $l_{1}$ with or to the right of $\left\langle s_{0}-1, v s_{0} \ominus 1\right\rangle$ ) and Final_bit $=0$ and no Final in Channel 2 .

\{Since $\left\langle s_{0}-1, v s_{0} \theta 1\right.$ ) was the last $I^{\prime}$ frame sent before retransmission was initiated through checkpointing, the Poll is either not outstanding or has been sent after $\left\langle s_{0}-1, v s_{0} \Theta 1\right\rangle$ was sent. $\}$

(e) Checkpoint_Cycle and Poll in Channel $_{1} \Rightarrow$ Poll is with or to the left of $\langle S-i, V S \ominus i\rangle$ where $i=V S \ominus V C S$.

\{Relates position of a checkpoint Poll in Channel $l_{1}$ to VCS.\}

\subsection{Image Protocol for $P_{2}$ to $P_{1}$ Data Transfer}

We now consider the function of one-way data transfer from $P_{2}$ to $P_{1}$. Two desirable properties of the HDLC protocol concerning this function may be stated as follows:

If $\operatorname{Mode}_{2}=$ Mode $_{1}=$ Open then

1. $\operatorname{Sink}_{1}[i]=$ Source $_{2}[i]$ for $0 \leq i<$ User_out $_{1}$

2. $0 \leq A_{2} \leq S_{2}<A_{2}+N$ 
The first property states that data is transferred in sequence; the second that the maximum number of outstanding data blocks (hence the minimum storage requirement) at $\mathrm{P}_{2}$ is $N-1$. These properties can be stated using only the variables Mode, Sink, and User_out at $\mathrm{P}_{1}$, and Mode, Source, $S$ and $A$ at $\mathrm{P}_{2}$. As in the case of the $\mathrm{P}_{1}$ to $\mathrm{P}_{2}$ data transfer, we can construct an image protocol using the variables Poll_Timer, $\$$ Poll_Timer, Poll_Retry_Count, VR, R, and Local_ RStatus at $\mathrm{P}_{1}$, and Final_bit, \$Response_Time, U_Response, User_in, VS, VA, VCS, Checkpoint_Cycle and Remote_RStatus at $\mathrm{P}_{2}$. (This image protocol is not well-formed.)

The images of message types sent by $P_{1}$ can be defined as follows. The image of message type (U, $P$, Command) is ( $\mathrm{U}^{\prime}, P$, Command). The image of ( $\mathrm{I}, P$, Data, NS, NR) is defined as $\left(\mathrm{I}^{\prime}, P, N R\right)$, where $\mathrm{I}^{\prime}$ is a new message type corresponding to an I frame in the HDLC protocol. The image of (S, $P, R$ Status, $N R$ ) is ( $\mathrm{S}^{\prime}, P, R$ Status $\left.N R\right)$.

Next we consider the images of the HDLC message types sent by $\mathrm{P}_{2}$. The image of (U, F, Response) is ( $\mathrm{U}^{\prime}, F$, Response). The image of (I, F, Data, NS, $N R)$ is $\left(\mathrm{I}^{\prime}, F\right.$, Data, NS), where $\mathrm{I}^{\prime}$ is a new message type corresponding to an $\mathrm{I}$ frame of the HDLC protocol. The image of $\left(\mathrm{S}^{\prime}, F, R\right.$ Status, $\left.N R\right)$ is $\left(\mathrm{S}^{\prime}, F\right)$, where $S^{\prime}$ is a new message type corresponding to an $S$ frame of the HDLC protocol.

Thus, in the image protocol, $\left(\mathrm{U}^{\prime}, P\right.$, Command $),\left(\mathrm{I}^{\prime}, P, N R\right)$ and $\left(\mathrm{S}^{\prime}, F\right.$, RStatus, $N R)$ are the message types sent by $\mathrm{P}_{1}$, and $\left(\mathrm{U}^{\prime}, F\right.$, Response), $\left(\mathrm{I}^{\prime}, F\right.$, Data, NS), and $\left(S^{\prime}, F\right)$ are the message types sent by $P_{2}$.

The events of $P_{1}$ and $P_{2}$ are shown in Tables $X$ and $X I$, respectively. The channel and time events of this image protocol are as in Tables IV and V. The initial state of this image protocol is the same as that of the HDLC protocol.

3.4.1. Safety Properties. For this image protocol, we have verified the desirable safety properties of the projected function. The following assertions, in conjunction with the $\mathrm{PF}$ assertions, are inductively complete and hence invariant. The notation used in these assertions is similar to the notation used in the assertions for the image protocol of $\mathrm{P}_{1}$ and $\mathrm{P}_{2}$ data transfer (with the role of $\mathrm{P}_{1}$ and $\mathrm{P}_{2}$, Channel $l_{1}$ and Channel $_{2}$, and Poll and Final interchanged).

The assertions $\mathrm{C} 1-\mathrm{C} 6$ listed below hold at all times. $\mathrm{C} 1-\mathrm{C} 5$ are concerned with conditions that hold during opening/closing of the data link; $\mathrm{C} 6$ is concerned with conditions of data transfer that hold when the data link is open.

C1. (a) Mode $_{1}=$ Open $\Rightarrow$ Mode $_{2}=$ Open and no U' frames in Channel and no $\mathrm{U}^{\prime}$ frames in Channel $\mathrm{C}_{2}$ and $U_{-}$Response = None

(b) Mode $_{1}=$ Closed $\Rightarrow$ Mode $_{2}=$ Closed and Channel Ch $_{1}$ is empty and $C$ hannel $l_{2}$ is empty and $U_{-}$Response $=$None

(c) $\left(\mathrm{I}^{\prime}, P, N R\right)$ or $\left(\mathrm{S}^{\prime}, P, R S t a t u s, N R\right)$ in Channel $_{1} \Rightarrow$ Mode $_{2}=$ Open.

C2. Poll_Timer $=$ Off $\Rightarrow U_{-}$Response $=$None and $\left(\right.$ Mode $_{2}=$ Open or Mode M $_{2}=$ Closed $)$.

C3. ( $\mathrm{U}^{\prime}, P$, Command) in Channel 1

$\Rightarrow$ Channel $_{1}$ satisfies (U', 1, Command), [Old_Ack_Sequence]

and (Command $=\mathrm{SARM} \Rightarrow$ Mode $_{1}=$ Opening)

and (Command $=\mathrm{DISC} \Rightarrow$ Mode $_{1}=$ Closing) 
and $\left(\right.$ Mode $_{2}=$ Open or $M o d e_{2}=$ Closed $)$ and $U_{-}$Response $=$None and Channel $_{2}$ satisfies [Old_Info_Sequence].

C4. $U_{-}$Response $\neq$None $\Rightarrow$ Final_bit $=1$

and Channel $_{1}$ is empty and Channel $l_{2}$ satisfies [Old_Info_Sequence]

and $\left(U_{-}\right.$Response $=\mathrm{UA} \Rightarrow\left(\right.$ Mode $_{1}=$ Mode $_{2}=$ Opening

or Mode $_{1}=$ Mode $_{2}=$ Closing))

and $\left(U_{-}\right.$Response $=\mathrm{DM} \Rightarrow\left(\right.$ Mode $_{1}=$ Closing

and Mode $_{2}=$ Closed)).

C5. (U', F, Response) in Channel $\Rightarrow$ Channel $_{1}$ empty

and ((Channel $l_{2}$ satisfies (U', $\left.1, \mathrm{DM}\right),[$ Old_Info_Sequence]

and Mode $_{2}=$ Closed and Mode M $_{1}=$ Closing)

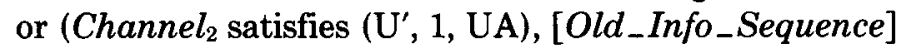

and Mode $_{2}=$ Closed and Mode Mlosing $_{1}$

or (Channel S $_{2}$ satisfies $\langle S-1, V S \ominus 1\rangle \ldots\langle 0,0\rangle$, (U', 1, UA),

[Old_Info_Sequence]

and Mode $_{2}=$ Open, $V A=A=0,0 \leq V S=S<N$,

Checkpoint_Cycle $=$ False, $U_{-}$Response $=$None

and Mode $_{1}=$ Opening)).

\{C5 supplies the initial condition for the next assertion, C6, to hold when the data link is opened at $P_{1}$.

C6. If Mode $_{1}=$ Open then $\left(\right.$ Mode $_{2}=$ Open and D1 and D2 and D3 and D4 and (D5 or D6)) holds, where D1-D6 are the assertions listed below.

D1. Source $[i]=\operatorname{Sink}[i]$ for $0 \leq i<U_{s e r}$ out $\leq R$.

D2. $A \leq R \leq S<A+N$.

D3. $A \bmod N=V A, S \bmod N=V S, R \bmod N=V R$.

D4. Checkpoint_Cycle $=$ True $\Rightarrow V S \ominus V A>V C S \ominus V A$.

D5. (a) Channel $l_{2}$ satisfies $\langle S-1, V S \ominus 1) \ldots\langle S-n, V S \ominus n\rangle$ where $0 \leq n \leq S-R$.

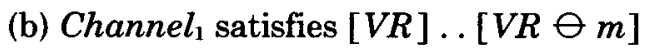
where $0 \leq m \leq R-A$.

(c) Checkpoint_Cycle and Final in Channel $_{2} \Rightarrow(n=0$ and VCS $=$ VS $\ominus 1)$ or ( $n>0$ and Final is with or to immediate left of $\langle S-i, V S \ominus i\rangle$ where $i=V S \ominus V C S$ )

or ( $n>0$ and Final is to right of $(S-n, V S \ominus n)$ and $n=(V S \ominus V C S)-1)$.

(d) Checkpoint_Cycle and Poll_Timer $=$ Off and $(V R \ominus V A \leq V C S \ominus V A) \Rightarrow S-n>R$.

(e) Checkpoint_Cycle and (Poll, NR) in Channel and $(N R \ominus V A \leq V C S \ominus V A) \Rightarrow N R=V R$ and $S-n>R$.

D6. (a) Channel $l_{2}$ satisfies $\langle S-1, V S \ominus 1\rangle \ldots\langle S-j, V S \ominus j\rangle$, $\left\langle s_{0}-1, v s_{0} \ominus 1\right\rangle \ldots\left\langle s_{0}-k, v s_{0} \ominus k\right\rangle$ 
where $j=S-A, A+N>s_{0}>s_{0}-k>R$, and $v s_{0}=s_{0} \bmod N$.

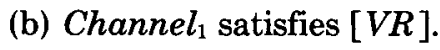

(c) $R=A$.

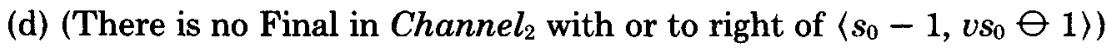
and Poll_ $_{-}$Timer $\neq$Off and no Poll in Channel . $_{1}$.

(e) Checkpoint_Cycle and Final in Channel $\Rightarrow$ Final is with $\langle S-j, V S \ominus j\rangle$.

\subsection{A Proposed Modification to HDLC}

We observed above that HDLC protocol cannot be considered to be well-structured, since it does not have well-formed image protocols that are significantly smaller than the HDLC protocol itself for all of its functions. We will now introduce a minor modification to the I message in the HDLC protocol. This modification allows us to obtain small well-formed image protocols for each of the one-way data transfer functions.

Our modification consists of adding an RStatus field to the HDLC I message type. In place of the message type (I, $P$, Data, $N S, N R$ ) sent by $\mathrm{P}_{1}$, we will use the message type (IM, $P$, Data, NS, NR, RStatus), where IM (standing for I modified) is the name of the message type. In place of the message type (I, $F$, Data, $N S, N R$ ) sent by $\mathrm{P}_{2}$, we will use the message type (IM, $F$, Data, NS, NR, RStatus). Note that the RStatus field can be implemented using a field of one bit.

The usage of this IM message type is similar to the usage of the HDLC I message type, except for the following difference. The $P$ field being set to 1 does not indicate any flow control information; instead, the RStatus field is used to convey flow control information exactly as in an $S$ frame. The events of this modified HDLC protocol system are shown in Tables XII and XIII. Note that the only difference between this modified HDLC protocol and the original HDLC protocol (Tables I and II) is that Send_I and Rec_I have been replaced by Send_ IM and Rec_IM, respectively. This modified HDLC protocol possesses small well-formed image protocols for each of its functions [16] and can be considered to be well-structured.

In particular, the image protocol obtained for connection management in Section 3.2 (Tables VI and VII) is still valid and well-formed (where $I^{\prime}$ is now the image of IM and S).

In the image protocol for one-way data transfer from $P_{1}$ to $P_{2}$, we have the same entity variables as before (Section 3.3). The message type $U$ is not changed in the image protocol (as before). The image of message types IM and S sent by $P_{1}$ are $I^{\prime}$ and $S^{\prime}$ respectively, as already defined. The message types IM and $\mathbf{S}$ sent by $P_{2}$ are both projected onto the same image message type $S$ (i.e., the Data and NS fields in IM are deleted). The events of $\mathrm{P}_{1}\left(\mathrm{P}_{2}\right)$ in the image protocol are exactly as shown in Table VIII (Table IX), except that Rec_I'(Send_I') is missing. This image protocol can easily be shown to be well-formed [16].

The image protocol for one-way data transfer from $P_{2}$ to $P_{1}$ can be similarly constructed. The entity variables of $P_{1}$ and $P_{2}$ in the image protocol are as defined 
in Section 3.4. The message types IM and $S$ sent by $P_{1}$ are both projected onto the same message type $\mathrm{S}$. The message types IM and $\mathrm{S}$ sent by $\mathrm{P}_{2}$ have the images $I^{\prime}$ and $S^{\prime}$ respectively as already defined. The events of $P_{1}\left(P_{2}\right)$ in the image protocol are exactly as shown in Table X (Table XI), except that Send_I' (Rec_I') is missing. This image protocol can easily be shown to be well-formed.

The connection management assertions obtained in Section 3.2.1, and the data transfer assertions obtained in Sections 3.3.1 and 3.4.1 continue to be invariant for the image protocols of this modified HDLC protocol. In addition, because these image protocols are well-formed, they are faithful to the modified HDLC protocol.

\section{CONCLUSION}

We have specified a version of the HDLC protocol using an event-driven process model, and verified it using the method of projections. The verification serves as a rigorous exercise in demonstrating the applicability of our method to the analysis of real-life protocols.

The HDLC protocol specified is based upon the Asynchronous Response Mode (ARM) of operation between two protocol entities, and includes all of its important features. It uses the basic repertoire of HDLC commands and responses (with the exception of the CMDR response), and includes the use of poll/final messages for checkpointing and connection management, timers for timeouts, cyclic sequence numbers, sliding windows of size $N$ for data transfers, and ready/ not ready messages for flow control.

The HDLC protocol has two characteristics found in most real-life communication protocols. First, the HDLC protocol is a time-dependent system, that is, HDLC operates under real-time constraints that are important not only for the protocol's performance efficiency but also for its correct logical behavior. Such time-dependent behavior cannot be handled by liveness assertions of the temporal logic variety. By including time variables and time events in our protocol model, we specify the HDLC time-dependent behavior in terms of safety assertions.

Second, HDLC is a multifunction protocol. It implements three distinguishable functions: connection management, and one-way data transfers between two protocol entities. Using the method of projections, we have constructed for each function an image protocol containing only those portions of the HDLC protocol that are needed to verify the desired correctness properties of that function. In each case, an inductively complete assertion implying the desired behavior was obtained.

Of the three image protocols obtained, only the connection management image protocol is well-formed. In order to construct a well-formed image protocol for one-way data transfer, almost the entire HDLC protocol has to be included in the image. This is due to dependencies in the two data transfer functions of HDLC. Thus, we say that the HDLC protocol as currently specified is not wellstructured. We then introduced a minor modification to the HDLC protocol, in the form of an additional one-bit flow control field in the HDLC I frame format. With this modification, small well-formed image protocols can be constructed for each of the HDLC functions of interest. Our modified version of HDLC can be considered as as well-structured protocol. 


\section{APPENDIX A}

Proof of PF Assertions.

Initially, Poll_bit $=0$, Poll_$_{-}$Timer $=$Off, Final_bit $=0, \$$ Response $\_$Time $=$Off, and the channels are empty. Hence, PF2 holds nonvacuously, while the rest of the PF assertions hold vacuously. We will next show that the PF assertions are true after the occurrence of any event, provided that they are true before the event occurrence. This will then establish the PF assertions as a system invariant.

We first introduce some notation that is used in the proof. The name of a variable will be used to denote its value before the occurrence of an event. We will now consider each of the events of the image protocol system and show that the $\mathrm{PF}$ assertions are not violated by any of them.

Entity events of $P_{1}$. User_req_conn and User_req_disc do not affect any of the PF assertions.

In order to send a Poll (using either Send_U' or Send_I'), the condition Poll_ bit $=1$ must hold. From PF1 and PF2, this means that there is no Poll in Channel $_{1}$, no Final in Channel 2 , and Final_bit $=0$. After the event, Poll_Timer $=\$ P o l L_{-}$Timer $=0$, and there is a single (Poll, $\$ A g e$ ) in Channel ${ }_{1}$ with $\$ A g e$ $=0$ (Poll is either a $\mathrm{U}^{\prime}$ or $\mathrm{I}^{\prime}$ frame). This establishes PF3 after the event. The other $\mathrm{PF}$ assertions are vacuously true.

Sending a non-Poll message does not affect the PF assertions.

When a Final message (either a $U^{\prime}$ or an $I^{\prime}$ frame) is received, from PF5 we have the following: there is no Poll in Channel $_{1}$, Final_bit $_{-} 0$ and Channel $_{2}$ has exactly one Final. Hence after the event occurrence, PF2 holds nontrivially, while the other PF assertions hold vacuously.

Reception of a non-Final message does not affect the PF assertions.

Request_Poll event makes PF1 hold nontrivially. The other PF assertions are not affected and continue to hold.

Poll_Timeout event can occur only when Poll_Timer $\geq$ PollTimeoutValue. Since PollTimeoutValue $>1+(1+a)\left(\right.$ MaxDelay $_{1}+$ MaxResponseTime + MaxDelay $_{2}$ ), and (from the accuracy axiom) $\mid$ Poll_Timer $-\$$ Poll_Timer $\mid \leq$ $1+a \$$ Poll_Timer, we have $\$$ Poll_Timer $>$ MaxDelay $_{1}+$ MaxResponseTime + MaxDelay $_{2}$. If any one of PF3, PF4 or PF5 held nonvacuously before the Poll_ Timeout event occurrence, that would imply either that channel $C_{1}$ contains an over-age message ( $\$$ Age $>$ MaxDelay $y_{1}$ in PF3), or that entity $\mathrm{P}_{2}$ waits too long to send a Final (\$Response _Time > MaxResponseTime in PF4), or that channel C2 contains an over-age message ( $\$$ Age $>$ MaxDelay $_{2}$ in PF5). Because of the local time axioms of these components, none of this can occur. Hence, PF3, PF4 and PF5 will hold vacuously before the Poll_Timeout event. Hence PF2 holds nonvacuously after the Poll_Timeout event.

Entity events of $\mathrm{P}_{2}$. The reception of Poll (either a $\mathrm{U}^{\prime}$ or an $\mathrm{I}^{\prime}$ frame) means that PF3 held nonvacuously. Also, from the local time axiom for channel $\mathrm{C}_{1}$ we have that $\$$ Poll_Timer $=\$ A g e \leq$ MaxDelay $y_{1}$. After the reception of the Final, Final_bit $=1$ and $\$$ Response $\_$Time $=0$. Hence PF4 holds nonvacuously after the event. The other PF assertions hold vacuously.

In order to send a Final message, Final_bit must equal 1. Hence PF4 holds nonvacuously. Also, from the local time axiom of entity $\mathrm{P}_{2}$ (\$Response_Time $\leq$ 
MaxResponseTime), we have that $\$$ Poll_Timer $\leq$ MaxDelay $_{1}+$ MaxResponse Time. After the event occurrence, Final_bit $=0, \$$ Response _ Time $_{-}$ $=$ Off, and a (Final, $\$ A g e$ ) message with $\$ A g e=0$ has been placed in the Channel $_{2}$. Hence, PF5 holds nonvacuously after the event occurrence, while the other PF assertions hold vacuously.

Reception of a non-Poll message or sending of a non-Final message does not affect the PF assertions.

Channel Events. Recall that the only channel event we have is a loss of the first message in any channel. The only effect of this message loss on the PF assertions is to transform PF3 (or PF4) from nonvacuously true to vacuously true. Hence the channel events do not violate the PF assertions.

Time Events. The occurrence of Poll_Timer_Tick does not affect the PF assertions since Poll__Timer is never set to Off by this event. The occurrence of the global time event increments by 1 tick both the left and right hand sides of the expressions involving $\$$ Poll__Timer in PF3, PF4, and PF5. Hence, none of the $\mathrm{PF}$ assertions are violated by the time events. 
Table I. Events of Primary HDLC Entity $P_{1}$

\begin{tabular}{|c|c|c|}
\hline Event Name & Enabling Cond1tion & Action \\
\hline 1. User_req_conn & $\begin{array}{l}\text { Mode } \neq \text { Opening } \\
\text { and Mode } \neq \text { Closing }\end{array}$ & Mode := Openting \\
\hline 2. User_req_disc & Mode $=$ Open & Mode := Closing \\
\hline 3. User_puts_data & $\begin{array}{l}\text { Mode = Open } \\
\text { and (User_in-A<Sbufsize) }\end{array}$ & $\begin{array}{l}\text { (User places data in } \\
\text { Source[User_1n]) } \\
\text { User_1n }:=\bar{U}_{\text {ser_1n }}+1\end{array}$ \\
\hline 4. User_gets_data & $\begin{array}{l}\text { Mode = Open } \\
\text { and (R-User_out > } 0)\end{array}$ & $\begin{array}{l}\text { \{User extracts data block } \\
\text { from S1nk[User_out]\} } \\
\text { User_out := User_out }+1 ; \\
\text { if Local_RStatus }=\text { RNR } \\
\text { then Local_RStatus := RR }\end{array}$ \\
\hline 5. Send_U & $\begin{array}{l}\text { (Mode = Opening } \\
\text { or Mode = Closing) } \\
\text { and Poll_bit }=1\end{array}$ & $\begin{array}{l}\text { If Mode }=\text { Opening } \\
\text { then Command }:=\text { SARM; } \\
\text { if Mode }=\text { Closing } \\
\text { then Command }:=\text { DISC; } \\
\text { put(Channel } 1 \text { (U, } 1 \text {, Command)): } \\
\text { POLL_SENT }\end{array}$ \\
\hline 6. $\operatorname{Rec}_{-} \mathrm{U}$ & first $\left(\right.$ Channel $\left.1_{2}\right)=U$ & $\begin{array}{l}\text { get (Channel } 2,(U, R, \text { Response)): } \\
\text { If Response }=D M \\
\text { then Mode }:=\text { Closed; } \\
\text { if (Response }=\text { UA and Mode }=\text { Closing) } \\
\text { then Mode }:=\text { Closed: } \\
\text { If (Response }=\text { UA and Mode }=\text { opening) then } \\
\text { begin } \\
\text { Mode : = Open; } \\
\text { INITIALIZE_SEND VARIABLES: } \\
\text { INITIALIZE_REC_VARIABLES } \\
\text { end; } \\
\text { If } \mathrm{F}=1 \text { then FINAL_RECEIVED }\end{array}$ \\
\hline 7. Poll_Timeout & Poll_T1mer $\geq$ PolltimeoutValue & $\begin{array}{l}\text { Reset(Poll_Timer, Off): } \\
\text { if Poll_Retry_Count < MaxRetryCount } \\
\text { then Poll_Retry_Count:=Poll_Retry_Count }+1 \\
\text { else Mode }:=\text { LinkFallure }\end{array}$ \\
\hline 8. Request_Poll & Poll_Timer $=01 f$ & Poll_b1t := 1 \\
\hline 9. Send_I & $\begin{array}{l}\text { Mode }=\text { Open } \\
\text { and VSeVA }<N-1 \\
\text { and } S<U S e r 1 n \\
\text { and Remote RStatus = RR } \\
\text { and not(Poillbit = } 1 \\
\text { and Local_RStatus = RNR) }\end{array}$ & $\begin{array}{l}\left.\text { put(Channe } 1_{1} \text {. (I,Poll_b1t, Source }[S], V S, V R\right) \text { ): } \\
\text { vs := vS } \theta 1: S:=S+1: \\
\text { If Poll_b1t }=1 \text { then } \\
\text { begin } \\
\text { CHECKPOINT_SENT: } \\
\text { POLL_SENT } \\
\text { end }\end{array}$ \\
\hline 10. Send_S & Mode $=$ Open & $\begin{array}{l}\text { put(Channel }, \text { (S,Poll_bit, Local_RStatus, VR)) } \\
\text { if Poll_bit }=1 \text { then } \\
\text { begin } \\
\text { CHECKPOINT_SENT; } \\
\text { POLL_SENT - } \\
\text { end }\end{array}$ \\
\hline 11. Rec_I & first $\left(\right.$ Channel $\left.1_{2}\right)=I$ & 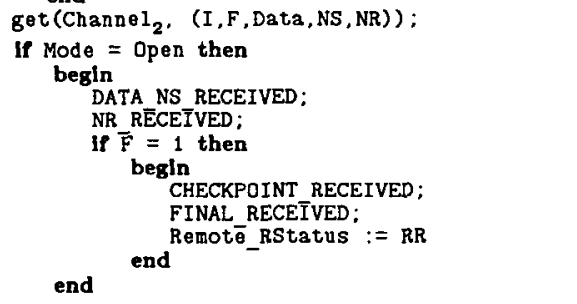 \\
\hline 12. Rec_S & first $\left(\right.$ Channel $\left.l_{2}\right)=s$ & $\begin{array}{l}\text { get }(\text { Channel } 2,(S, F, \text { RStatus, NR })) \text {; } \\
\text { If Mode }=\text { Open then } \\
\text { begin } \\
\text { Remote RStatus := RStatus: } \\
\text { NR RECEIVED; } \\
\text { if } \bar{F}=1 \text { then } \\
\text { begin } \\
\text { CHECKPOINT RECEIVED; } \\
\text { FINAL_RECEIVED } \\
\text { end }\end{array}$ \\
\hline
\end{tabular}


Table II. Events of Secondary HDLC Entity $\mathbf{P}_{2}$

\begin{tabular}{|c|c|c|}
\hline Event Name & Enabling Condition & Action \\
\hline 1. User_puts_data & $\begin{array}{l}\text { Mode = Open } \\
\text { and (User_in-A<SbufSize) }\end{array}$ & $\begin{array}{l}\text { \{User places data block in Source[User_in]\} } \\
\text { User_1n }:=\text { User_in }+1\end{array}$ \\
\hline 2. User_gets_data & $\begin{array}{l}\text { Mode }=\text { Open } \\
\text { and ( } R \text { - User_out }>0 \text { ) }\end{array}$ & $\begin{array}{l}\text { \{User extracts data block from S1nk[User_out]\} } \\
\text { User_out := User_out }+1 ; \\
\text { if Local RStatus = RNR } \\
\text { then Local_RStatus : = RR }\end{array}$ \\
\hline 3. Rec_U & first $\left(\right.$ Channel $\left.1_{1}\right)=U$ & 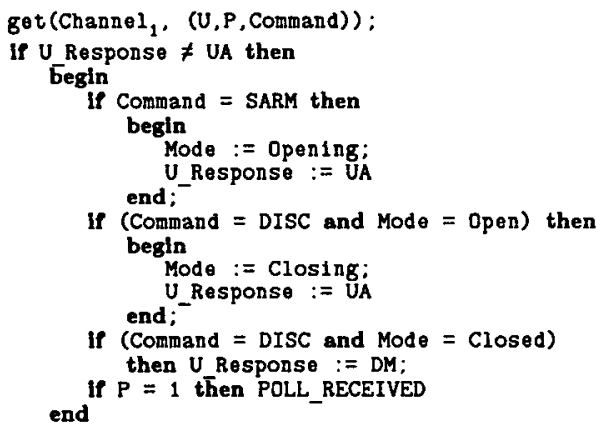 \\
\hline 4. Send_U & U_Response $\neq$ None & $\begin{array}{l}\text { put(Channel }{ }_{2} \text {. (U,Final_bit,U_Response)): } \\
\text { U Response }:=\text { None: } \\
\text { If Mode = Closing then Mode }:=\text { Closed: } \\
\text { If Mode }=\text { Opening then } \\
\text { begin } \\
\text { Mode }:=\text { Open: } \\
\text { INITIALIZE_SEND_VARIABLES: } \\
\text { INITIALIZE_REC_VARIABLES } \\
\text { end; } \\
\text { if Final_bit }=1 \text { then FINAL_SENT }\end{array}$ \\
\hline 5. Send_I & $\begin{array}{l}\text { Mode }=\text { Open } \\
\text { and VSeVA<N-1 } \\
\text { and } S<\text { User in } \\
\text { and Remote_RStatus = RR } \\
\text { and not(F1nal_bit = } 1 \\
\text { and Local_RStatus = RNR) }\end{array}$ & 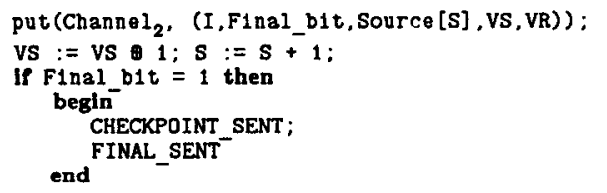 \\
\hline 6. Send_s & Mode $=$ Open & $\begin{array}{l}\text { put(Channel }{ }_{2} \text { (S,Final_bit,Local_RStatus, VR)): } \\
\text { if Final_bit }=1 \text { then } \\
\text { begin } \\
\text { CHECKPOINT_SENT; } \\
\text { FINAL_SENT } \\
\text { end }\end{array}$ \\
\hline 7. Rec_I & Plrst (Channel 1$)=I$ & 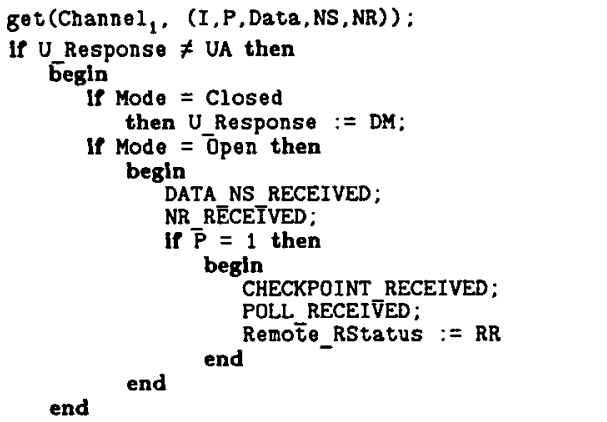 \\
\hline 8. Rec_S & first $($ Channel $)=S$ & 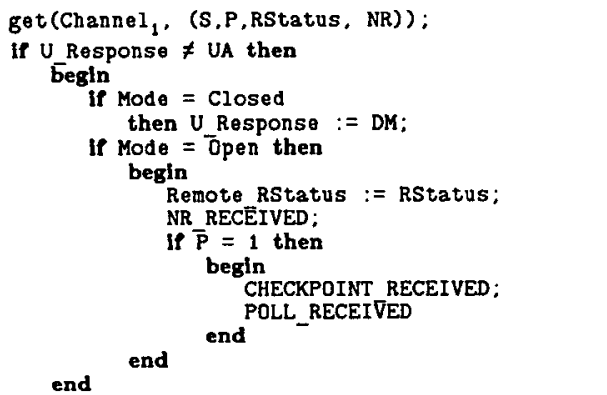 \\
\hline
\end{tabular}


Table III. Details of Code Segments Used in Tables

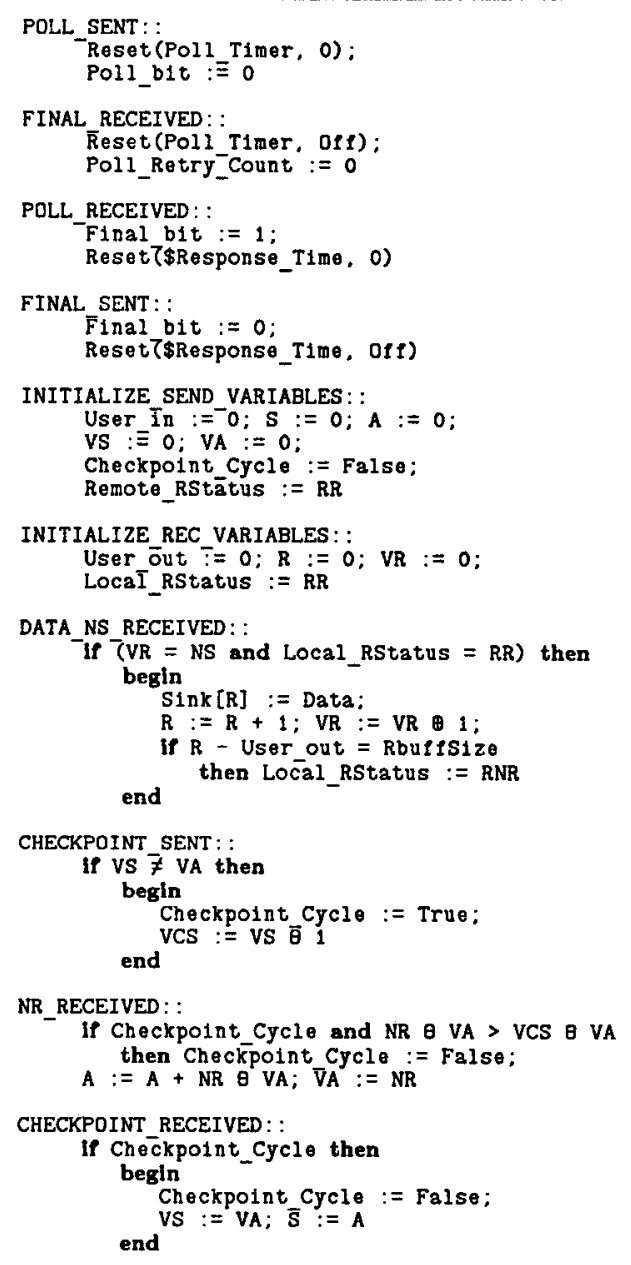

Table IV. Events of Channel $C_{i}$ for the Protocol System

\begin{tabular}{lll}
\hline Event Name & Enabiling Condition & Action \\
Message_Loss & Channel $_{1}$ is not empty & Delote the first message in Channel 1 \\
\hline
\end{tabular}

Table V. Time Events for the Protocol System

\begin{tabular}{|c|c|c|}
\hline Event Name & Enabling Condition & Action \\
\hline 1. Poll_Timer_Tick & $\begin{array}{c}\text { (Poll_Timer - \$Poll_Timer) } \\
\leq \text { a (\$Poll_Timer) }\end{array}$ & Age (Poll_Timer) \\
\hline \multirow[t]{3}{*}{ 2. Global_Tick } & $\begin{array}{l}\text { (\$Poll Timer - Poll Timer) } \\
\text { and }(\$ \bar{R} \text { a (\$Poll Timers }) \\
\text { anse T1me }\end{array}$ & $\begin{array}{l}\text { Age (\$POL__Timer): } \\
\text { Age (\$Response } T \text { ime): }\end{array}$ \\
\hline & $\begin{array}{c}\text { and (ail ages in Channel } \\
\text { ( MaxDelay, }\end{array}$ & Age (all ages in Channel $)$ : \\
\hline & $\begin{array}{l}\text { and (all ages in Channel } \\
\left.<\operatorname{MaxDelay}_{2}\right)\end{array}$ & Age (all ages 1n Channel ${ }_{2}$ ) \\
\hline
\end{tabular}


Table VI. Events of $P_{1}$ in the Image Protocol for Connection Management

\section{Event Name}

1. User_req_conn

2. User_reqdisc

3. Send_U'

4. Rec_U.

5. Poll_Timeout

6. Request_Poll

7. Send_I.

8. Rec_I'

Enabling Condition
(same as in Table 1)
(same as in Table 1)
(same as in Table 1)

first (Channel $\left.)_{2}\right)=U^{\prime} \quad \operatorname{get}\left(\right.$ Channel $_{2}$, (U', F, Response)):

If Response $=\mathrm{DM}$

then Mode := Closed:

If (Response=UA and Mode=Closing)

then Mode := Closed:

if (Response=UA and Mode=0pening)

then Mode := Open;

if $F=1$ then FINAL_RECEIVED

(same as in Table 1)

(same as in Table 1)

Mode $=$ Open

put (Channe 1, (I', Poll_bit)):

If Poll_bit $=1$ then POLL_SENT

get (Channel,$(I, F))$;

If Mode $=$ Open then

If $\mathrm{F}=1$ then FINAL_RECEIVED

Table VII. Events of $P_{2}$ in the Image Protocol for Connection Management

\begin{tabular}{|c|c|c|}
\hline Event Name & Enabling Condition & Action \\
\hline 1. Rec_U' & \multicolumn{2}{|c|}{ (same as in Table 2) } \\
\hline 2. Send_U' & U_Response $\neq$ None & $\begin{array}{l}\text { put (Channel }{ }_{2} \text { (U'Final_bit,U_Response)) } \\
\text { U Response := None: } \\
\text { if Mode }=\text { Closing } \\
\text { then Mode }:=\text { Closed; } \\
\text { If Mode }=\text { Opening } \\
\text { then Mode := Open: } \\
\text { if Final bit }=1 \\
\text { then FINAL_SENT }\end{array}$ \\
\hline 3. Send_I. & Mode $=$ Open & $\begin{array}{l}\left.\text { put (Channel })_{2}\left(I^{\prime}, \text { Final_bit }\right)\right) \text { : } \\
\text { If Final_b1t }=1 \text { then FINAL_SENT }\end{array}$ \\
\hline 4. Rec_I' & Iirst $\left(\right.$ Channel $\left.1_{1}\right)=I$. & $\begin{array}{l}\text { get (Channel },(I \cdot, P)) \text {; } \\
\text { If U Response } \neq \text { UA then } \\
\text { begin } \\
\text { if Mode }=\text { Closed } \\
\text { then U_Response }:=\text { DM: } \\
\text { if (Mode }=\text { Open and } P=1) \\
\text { then POLL_RECEIVED }\end{array}$ \\
\hline
\end{tabular}


Table VIII. Events of $P_{1}$ in the Image Protocol for the HDLC $P_{1}$ to $P_{2}$ Data Transfer

\begin{tabular}{|c|c|c|}
\hline Event Name & Enabling Condition & Action \\
\hline 1. User_req_conn & (same as in & Table 1) \\
\hline 2. User_req_dise & (same as $1 \mathrm{n}$ & Table 1) \\
\hline 3. User_puts_data & (same as $1 n$ & Table 1) \\
\hline 4. Send_U' & (same as in & Table 1) \\
\hline 5. Rec_U & first $\left(\right.$ Channel $\left.{ }_{2}\right)=U^{\prime}$ & 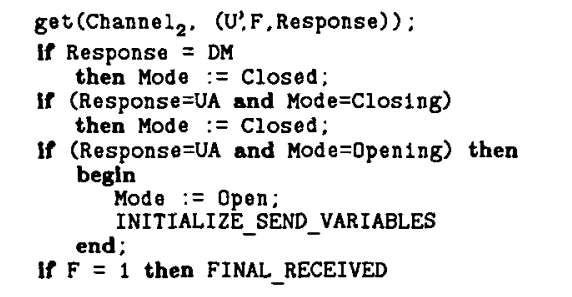 \\
\hline 6. Poll_Timeout & (same as in & Table 1) \\
\hline 7. Request_Poll & (same as in & Table 1) \\
\hline 8. Send_I' & $\begin{array}{l}\text { Mode }=\text { Open } \\
\text { and VSeVA }<N-1 \\
\text { and } S<\text { User in } \\
\text { and Remote_RS̄tatus = RR }\end{array}$ & 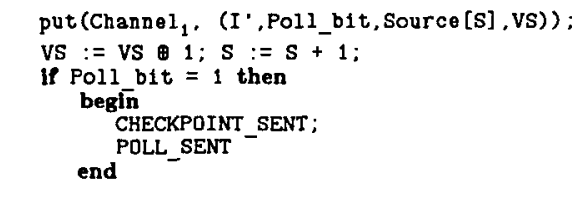 \\
\hline 9. Send_S' & 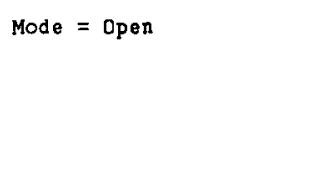 & $\begin{array}{l}\text { put (Channel, },(S \cdot, \text { Poll_bit })) \\
\text { If Poll_bit }=1 \text { then } \\
\text { begin } \\
\text { CHECKPOINT_SENT: } \\
\text { POLL_SENT } \\
\text { end }\end{array}$ \\
\hline 10. Rec_I. & first $\left(\right.$ Channel $\left.1_{2}\right)=I$ & 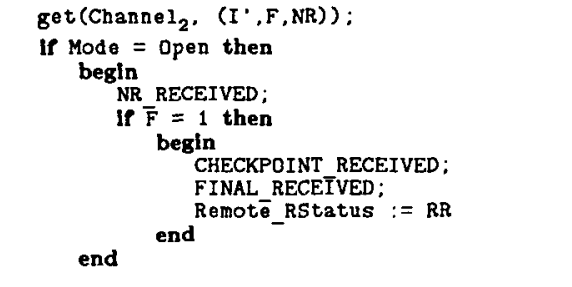 \\
\hline 11. Rec_s' & (same as in & Table 1) \\
\hline
\end{tabular}


Table IX. Events of $P_{2}$ in the Image Protocol for the HDLC $P_{1}$ to $P_{2}$ Data Transfer

\begin{tabular}{|c|c|c|}
\hline Event Name & Enabling Condition & Action \\
\hline 1. User_gets_data & (same as in & ble 2) \\
\hline 2. Rec_U' & (same as in & tble 2) \\
\hline 3. Send_U' & U_Response $\neq$ None & $\begin{array}{l}\text { put (Channel }{ }_{2} \text { (U',Final_bit, U Response)); } \\
\text { U Response := None: } \\
\text { If Mode = Closing } \\
\text { then Mode = Closed: } \\
\text { If Mode = Opening then } \\
\text { begin } \\
\text { Mode : = Open: } \\
\text { INITIALIZE_SEND_VARIABLES } \\
\text { end; } \\
\text { if Final_bit }=1 \text { then FINAL_SENT }\end{array}$ \\
\hline 4. Send_I. & $\begin{array}{l}\text { Mode }=\text { open } \\
\text { and not (Final_bit }=1 \\
\text { and Local_RStatus=RNR) }\end{array}$ & $\begin{array}{l}\left.\text { put(Channel },\left(I^{*}, \text { Final_bit.VR }\right)\right) \\
\text { if Final_b1t }=1 \text { then FINAL_SENT }\end{array}$ \\
\hline 5. Send_s' & Mode $=$ Open & $\begin{array}{l}\text { put(Channel }, \quad(S, \text { Final_bit,Local_RStatus, VR)) } \\
\text { if F1nal_bit }=1 \text { then FINAL_SENT }\end{array}$ \\
\hline 6. Rec_I' & first $\left(\right.$ Channel $\left.l_{1}\right)=I$. & $\begin{array}{l}\left.\text { get (Channel },\left(I^{\prime}, P, \text { Data, NS }\right)\right) ; \\
\text { If U Response } \neq \text { UA then } \\
\text { begin } \\
\text { if Mode = Closed } \\
\text { then U Response := DM; } \\
\text { If Mode = Open then } \\
\text { begln } \\
\text { DATA_NS_RECEIVED; } \\
\text { if } P=1 \text { then POLL_RECEIVED } \\
\text { end }\end{array}$ \\
\hline 7. Rec_s' & first $\left(\right.$ Channel $\left.{ }_{1}\right)=s^{\prime}$ & $\begin{array}{l}\left.\text { get (Channel },\left(S^{\cdot}, P\right)\right) \text { : } \\
\text { If U Response } \neq \text { UA then } \\
\text { begin } \\
\text { if Mode }=\text { Closed } \\
\text { then UResponse }:=\text { DM; } \\
\text { if Mode }=\text { Open and } P=1 \\
\text { then POLL_RECEIVED } \\
\text { end }\end{array}$ \\
\hline
\end{tabular}


Table X. Events of $P_{1}$ in the Image Protocol for the HDLC $P_{2}$ to $P_{1}$ Data Transfer

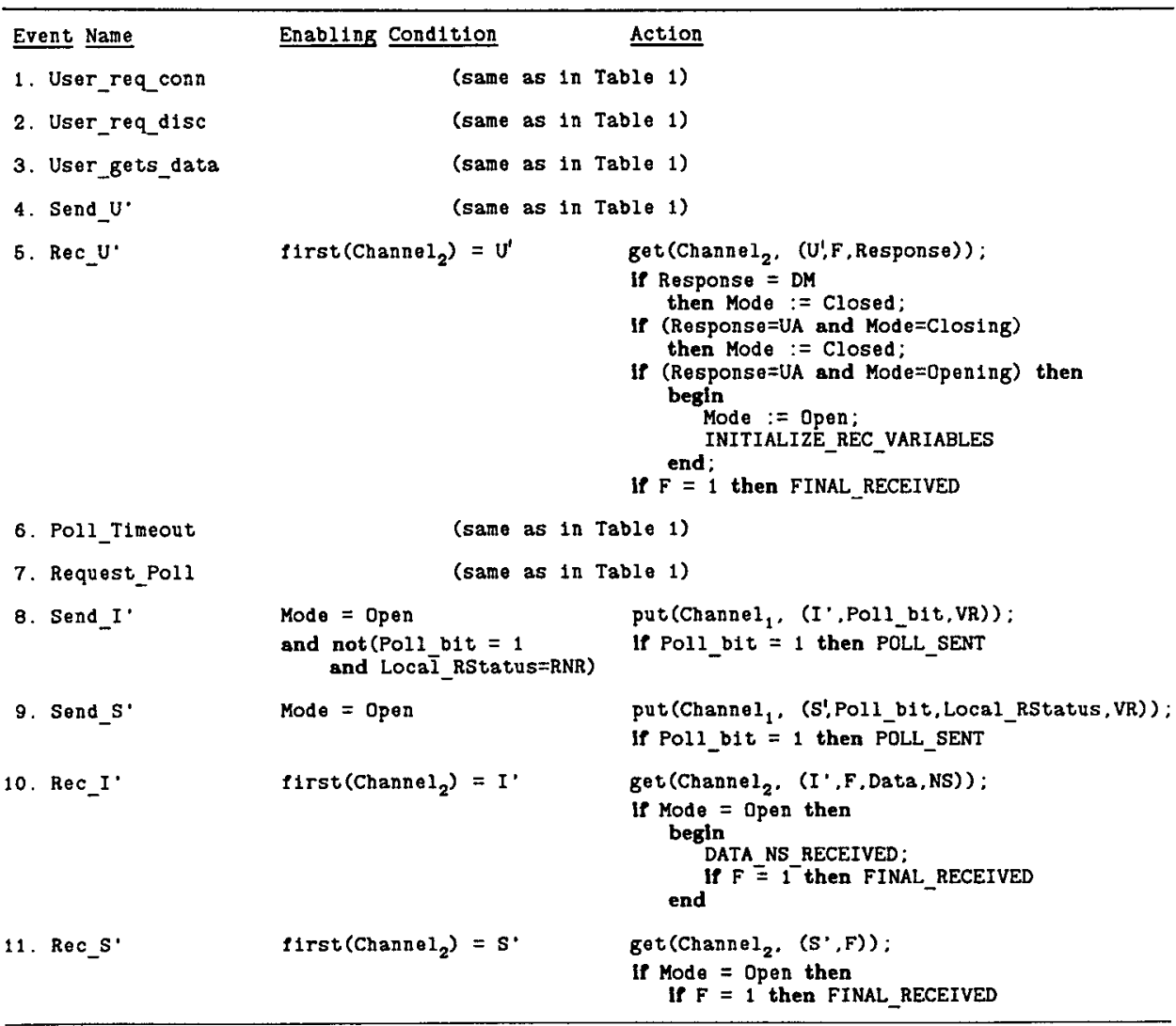


Table XI. Events of $P_{2}$ in the Image Protocol for the HDLC $P_{2}$ to $P_{1}$ Data Transfer

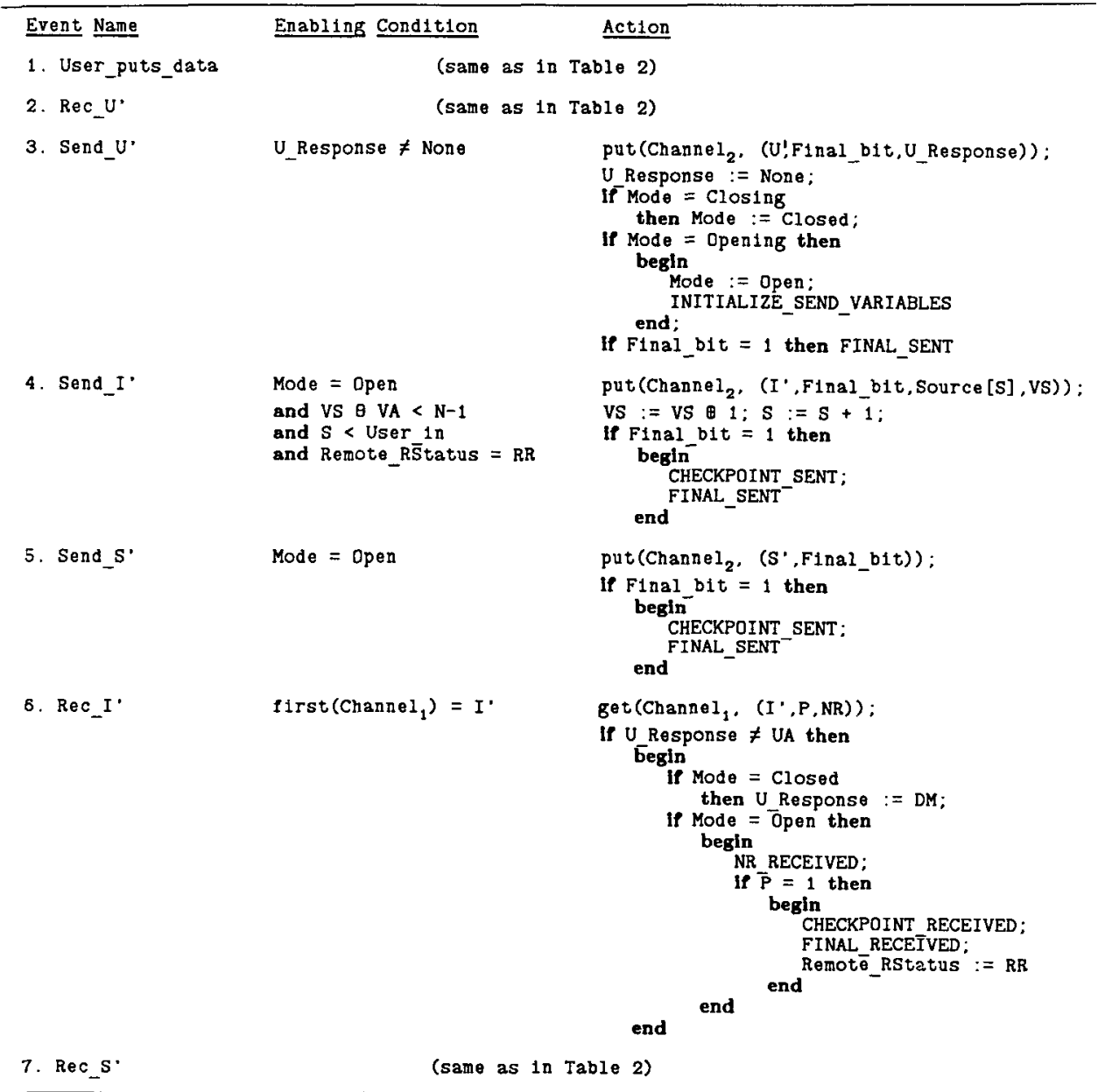


Table XII. Events of $P_{1}$ in the Modified HDLC Protocol

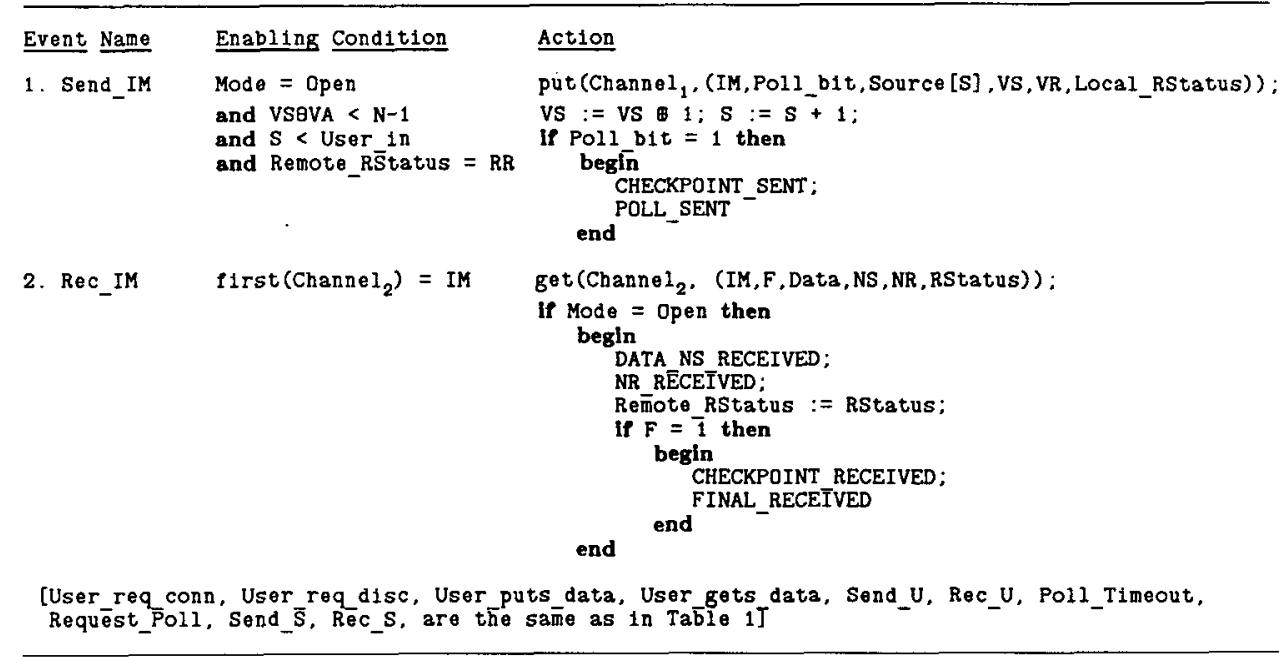

Table XIII. Events of $\mathrm{P}_{2}$ in the Modified HDLC Protocol

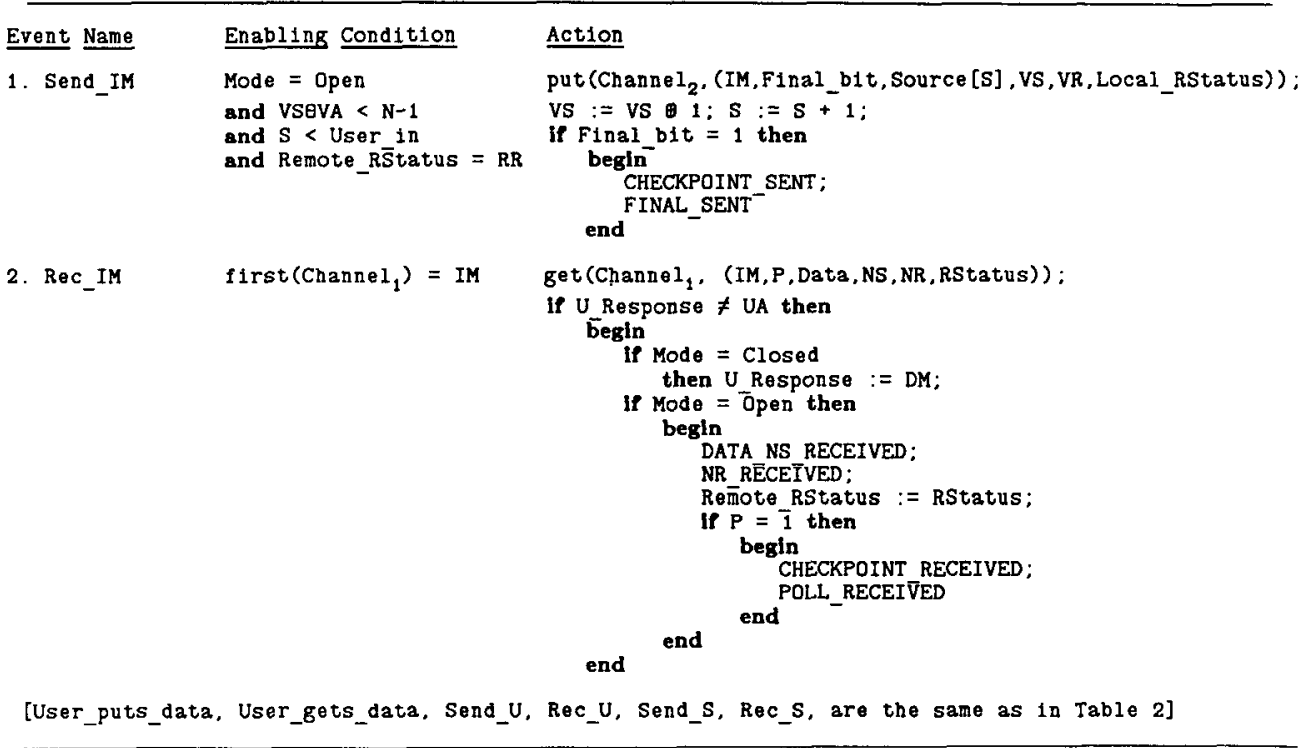

\section{REFERENCES}

1. Bochmann, G.V. Finite state description of communication protocols. Comput. Networks 2, (Oct. 1978), 361-372.

2. Bochmann, G.V., AND ChUng, R.J. A formalized specification of HDLC classes of procedures. In Conf. Rec. National Telecommunication Conference, (Los Angles, Dec. 1977), IEEE, New York, pp. 519-530. 
3. BochmanN, G.V., AND C.A. Sunshine, Formal methods in communication protocol design. IEEE Trans. Commun. COM 28, 4 (April 1980), 624-631.

4. BRAND, D. AND Joyner, W.H. Verification of HDLC. IEEE Trans. Commun. COM 30, 5 (May 1982), 1136-1142.

5. Diskstra, E.W. A Discipline of Programming. Prentice-Hall, Englewood, Cliffs N.J., 1976.

6. HAILPERN, B.T. AND OWICKI, S.S. Verifying network protocols using temporal logic. Tech. Rep. 192, Computer Systems Laboratories, Stanford University, Stanford, Calif., June, 1980.

7. International Standards Organization. Data Communincations-HDLC ProceduresFrame Structure. Ref. No. ISO 3309, International Standards Organization, Geneva, Switzerland, 1979.

8. International Standards Organization. Data Communications-HDLC Procedures-Elements of Procedures. Ref. No. ISO 4335, International Standards Organization, Geneva, Switzerland, 1979.

9. International Standards Organization. Data Communications-HDLC Unbalanced Classes of Procedures. Ref. No. ISO 6159, International Standards Organization, Geneva, Switzerland, 1980.

10. KUROSE, J. The specification and verification of a connection establishment protocol using temporal logic. In Proc. 2nd Int. Workshop on Protocol Specification, Testing and Verification, (Idyllwild, Ca., May 17-20, 1982), IFIP, New York, pp. 43-62.

11. Lam, S.S. and Shankar, A.U. Protocol projections: A method for analyzing communication protocols. In Conf. Rec. National Telecommunications Conference, (New Orleans, Nov. 1981), IEEE, New York.

12. LAM, S.S. AND ShankaR, A.U. Verification of communication protocols via protocol projections. In Proc INFOCOM '82, (Las Vegas, March 30-April 1, 1982), IEEE, New York, pp. 229-237.

13. Lam, S.S., And Shankar, A.U. Protocol Verification via Projections. Tech. Rep. 207, Dept. of Comput. Sci., Univ. of Texas at Austin, Aug., 1982. To appear in IEEE Trans. Softw. Eng.

14. Razouk, R. Modeling X.25 using the graph model of behavior. In Proc. 2nd Int. Workshop on Protocol Specification, Testing and Verification, (Idyllwild, Ca., May 17-20, 1982). IFIP, New York, pp. 197-214.

15. Schwartz, R.L. And Mellifar-Smith, P.M. From state machines to temporal logic: Specification methods for protocol standards. IEEE Trans. Commun. COM-30, 12 (Dec. 1982), 2486-2496.

16. Shankar, A.U. Analysis of communication protocols via protocol projections. Ph.D. thesis, Dept. of Electrical Engineering, University of Texas at Austin, Austin, Tx., December, 1982.

17. Shankar, A.U. AND Lam, S.S. On time-dependent communication protocols and their projections. In Proc. 2nd Int. Workshop on Protocol Specification, Testing and Verification, (Idyllwild, Ca., May 17-20, 1982), IFIP, New York, pp. 215-235.

18. Shankar, A.U. and Lam, S.S. An HDLC Protocol Specification and its Verification using Image Protocols. Tech. Rep. 212, Dept. of Computer Sciences, University of Texas at Austin, September, 1982 (first version).

19. Shankar, A.U. AND LAM, S.S. Specification and verification of an HDLC protocol with ARM connection management and full-duplex data transfer. In Proc. ACM SIGCOMM '83 Symposium, (Austin, Tx., March 8-9, 1983), ACM, New York, pp. 38-48.

20. Shankar, A.U. And Lam, S.S. Application of Projections to a Structured Model of Communication Protocols. Tech. Rep. 214, Dept. of Computer Sciences, University of Texas at Austin, 1983 (in preparation).

21. SLOAN, L. Mechanisms that enforce bounds on packet lifetimes. Presented at ACM SIGCOMM '83 Symposium, University of Texas at Austin, March 1983. ACM Trans. Comput. Syst. 1, 4 (Nov. 1983), 311-330 (this issue).

22. Stenning, N.V. A data transfer protocol. Comput. Networks 1 (Sept. 1976), 99-110.

23. ZimmermanN, H., OSI Reference Model- The ISO model of architecture for open systems interconnection. IEEE Trans. Commun. COM-28, 4 (April 1980), 425-432.

Received September 1982; revised May 1983, accepted July 1983 